\title{
Classical logic as Limit Completion
}

\author{
Stefano Berardi \\ http://www.di.unito.it $/{ }$ stefano \\ Università di Torino
}

\begin{abstract}
We define a constructive model for $\Delta_{2}^{0}$-maps, that is, maps recursively definable from a map deciding the halting problem. Our model refines existing constructive interpretation for classical reasoning over one-quantifier formulas: it is compositional (Modus Ponens is interpreted as an application) and semantical (rather than translating classical proofs into intuitionistic ones, we define a mathematical structure intuitionistically validating Excluded Middle for one-quantifier formulas).
\end{abstract}

Iniziato a Torino (Italia), il 24 Gennaio 2001. Ultimo salvataggio: November 26, 2004.

Acknowledgement. This paper comes out of a collection of notes taken in preparation of a short course in Kyoto, 7-19 January 2001. Such course would never have been possible without all the support and suggestions coming from Prof. Susumu Hayashi of Kobe University, and all people of the Proof Animation project. To them it goes my warmest gratitude. I thank also Prof. Yohji Akama, of Tohoku University, for proof-checking an earlier version of this paper, and for many valuable comments.

\section{Introduction}

A topological model for classical reasoning. Let $N$ denote the set of nonnegative integers. In our model, we define a completion of $N$, quite similar to the topological completion defining the set $R$ of Real numbers out of the set $Q$ of Rational numbers. As we all know very well, some converging (i.e., Cauchy) succession over $Q$ has no limit. In order to have a limit for all converging succession over $Q$, we consider the set of converging succession over $Q$ themselves as a new class of individual, the set $R$ of Real Numbers. Rational numbers are identified with constant successions over $Q$. In much the same way, since some converging succession over $N$ has no computable result, we consider the set of converging succession to $N$ as a new class of individual, and we denote it by $N^{*}$. The original elements of $N$ are identified with constants successions. All convergent successions over $N$ now have some computable result in $N^{*}$. From a classical viewpoint, $N^{*}$ is nothing but $N$, because each limit may be identified with its integer result. From a constructive viewpoint, several maps which were not computable over $N$ are computable over $N^{*}$ (but with a result in $N$, no more in $N$ ). Fix any two element subset $\mathcal{B}=\{\mathrm{tt}, \mathrm{ff}\}$ of $N$, and any total recursive $f: N^{2} \rightarrow \mathcal{B}$. Typical maps computable over $N^{*}$, but 
not computable over $N$, are the " $\mathbf{s}_{f}$ " map, defined by $\mathbf{s}_{f}(x)=$ tt if $f(x, y)=\mathrm{tt}$ for some $y$, and $\mathbf{s}_{f}(x)=\mathrm{ff}$ if $f(x, y)=\mathrm{ff}$ for all $y$. A " $\mathbf{s}_{f}$ " map decides solvability in $y$ of integer equations $f(x, y)=$ tt (one of the many formulations of the so-called Halting Problem, a problem without recursive solution).

Starting from this consideration, we build a purely intuitionistic (i.e., not using Excluded Middle at all) model of recursive maps + " $\mathbf{s}_{f}$ " maps: more precisely, of all $\Delta_{2}^{0}$-maps. We only need some minor shifts in definition: convergence of successions and equality between successions have to be reformulated. New definitions are classically equivalent to the more traditional ones, but from a constructive viewpoint they are more significant. Any classical reasoning using only the existence of the " $\mathbf{s}_{f}$ " map (essentially, any classical reasoning over one-quantifier formulas) may be reinterpreted as constructive reasoning in our model.

The main result we have for our topological model is: whenever an equation $g(x)=0$ (for $g$ recursive map) has some solution $x=l \in N^{*}$ (has some solution in $N$ ), then it has a solution $x=n \in N$ (has some solution in the ordinary sense). Besides, $n$ is not found by blind search through all integers, but it may be computed by using ideas from the proof of $g(l)=0$. In other words: by working in $N^{*}$, we may use non-recursive maps and one-quantifier classical reasoning in order to find effectively given solutions to recursive problems.

A Realization semantic for classical equational reasoning. By building over the top of our model of $\Delta_{2}^{0}$-maps, we may define an Heyting-like notion of "realizer" $\mathrm{r}$ for equations $l=m$ between limits. A "realizer" is a map making explicit the construction hidden in constructive reasoning. Unlike equational reasoning over integers, equational reasoning over limits hides some non-trivial construction, hence some non-trivial realizer. Eventually, we sketch an implementation of realizers, that is, an explicit constructive content for a classical proof using the " $\mathbf{s} f$ " maps.

All definition and proofs in this paper are purely intuitionistic. We use intuitionism as a tool to make explicit constructions usually hidden in classical reasoning.

Some Features of Classical Logic not considered in this paper. Often, a proof in Classical Logic hides a non-deterministic, parallel algorithm. We did not try, however, to represent such features of Classical Logic in $N^{*}$. There is also another relevant topic not included in this paper. In both Classical and Intuitionistic Arithmetic we may define, by a fixed point operator, maps which are convergent w.r.t. some evaluation strategy (say, call-by-name), yet divergent w.r.t. some other evaluation strategy (say, pure call-by value). In $N^{*}$ we will be able to represent different evaluation strategies for the same expression, yet we will only represent convergent evaluation strategies for any expression. In a forthcoming paper [1] we plan to show how non-determinism, parallelism, and divergent evaluation strategies may be represented in $N^{*}$, at the price of choosing a more general notion of convergence to define $N^{*}$.

This is the plan of the paper. We first will introduce completion $N^{*}$ of $N$, then a computational interpretation for equational reasoning on $N^{*}$. In 2 we introduce all intuitionistic concepts we need in the paper (wellfounded relations, direct sets and so forth). In 3 we define our model 
$N^{*}$ of limit reasoning over $N$. In 4 we characterize continuous maps on $N^{*}$ as $\Delta_{2}^{0}$-maps.

Eventually, in 5 we define a Realizability interpretation for equational reasoning. In 6 we use realizers to constructivize one simple classical theorem requiring Excluded Middle over one-quantifier formulas (over formulas without nested quantifiers).

\section{Some intuitionistic concepts}

In this section, we will introduce all ingredients we need for our completion of $N$ : direct sets, covering of $N$, sequences, successions, stationarity, limits, well-founded relations, change ordering, stability. The key notion of the paper will be the notion of stability (a kind of converge notion for limits).

Total recursive maps over subsets of $N$. Let $I, J \subseteq N$. A total recursive map $f: I \rightarrow J$ is any partial recursive map $f: N \rightarrow N$, total over $I \subseteq N$, and such that $f(I) \subseteq J$. This notion of total recursive may be generalized to maps of any arity.

Recursive Direct sets. $D$ is a direct set if $D$ is an ordered set, inhabited (there is some $d \in D$ ), and any two $d, e \in D$ have some upper bound $e^{\prime} \geq d, e$ in $D$. We will consider only direct sets $D$ which are recursive subsets of $N$, with a recursive ordering relation. We call them recursive direct sets.

Upper bound maps. We call upper bound map any total recursive map $j: D, D \rightarrow D$ picking, for any $d, e \in D$, some common upper bound $j(d, e) \geq d, e$ of them. All recursive direct sets have at least one upper bound map $j$, defined by $j(d, e)=$ the first $x \in N$ such that $x \in D$ and $x \geq d, e$. This map $j$ is total on $D$ because all $d, e \in D$ have some common upper bound in $D$, by definition of direct set. This map $j$ is recursive because the predicates $x \in D$ and $x \geq d, e$ are, by assumption, both recursive. We suppose that for each recursive directed set is fixed some upper bound map $j$. Usually, $j(d, e)$ will be the l.u.b. of $d, e$. For any $d_{1}, d_{2}, \ldots, d_{n} \in D$, we will write $j\left(d_{1}, d_{2}, \ldots, d_{n}\right)$ for $j\left(d_{1}, j\left(d_{2}, j\left(\ldots, d_{n}\right)\right)\right)$. $j\left(d_{1}, d_{2}, \ldots, d_{n}\right)$ is some common upper bound for $d_{1}, d_{2}, \ldots, d_{n}$ in $D$. The interpretation of $D$. $D$ represents a tree of possible computation states. When $d \leq e$, we say that the state $e$ is "a possible future" for the state $d$. We consider any increasing succession of computation states: $d_{0}<$ $d_{1}<d_{2}<d_{3}<\ldots$ as the history of some computation. For the purpose of defining a model of $\Delta_{2}^{0}$-maps, the example $D=N$ would suffice. In this simplified case, a canonical example of increasing succession of computation states is: $0<1<2<\ldots$. We choose, as upper bound map $j$ for $D=N$, the map $j(n, m)=\max (n, m)$. Another relevant example is $D=\mathcal{P}_{\text {fin }}(N)=\{$ finite subsets of $N\}$. We choose, as upper bound map $j$ for $D=\mathcal{P}_{\text {fin }}(N)$, the map $j(J, H)=J \cup H$. Only at the end of Part II we will consider a more subtle choice for $D$. A particular choice for $D$ may improve the description of the computations over $N^{*}$, and therefore the efficiency of the computational interpretation of classical proofs. However, different choices for D generate isomorphic models of $\Delta_{2}^{0}$-maps, as we are going to prove. In the rest of the paper the reader may assume that $D=\mathcal{P}_{\text {fin }}(N)$. 
Coverings of $N$. Let $D$ be a directed set. Then a covering structure of $N$ by $D$ is any family $\{\operatorname{Cov}(d)\}_{d \in D}$ of finite subsets of $N$ : (i) indexed by elements of $D$ in a recursive and increasing way, (ii) such that we may effectively find, for any $x \in N$, some element $\operatorname{Cov}(d)$ such that $x \in \operatorname{Cov}(d)$. Formally, a covering structure is a pair of recursive maps: Cov $: D \rightarrow \mathcal{P}_{\text {fin }}(N), \xi: N \rightarrow D$ satisfying the conditions: (i) Cov is weakly increasing: if $d \leq e$, then $\operatorname{Cov}(d) \subseteq \operatorname{Cov}(e)$; (ii) $x \in \operatorname{Cov}(\xi(x))$ for all $x \in N$. When a covering structure by $D$ is given, we say " $D$ covers $N$ ". When $x \in \operatorname{Cov}(d)$, we say that $d$ covers $x$. Intuitively, if we have a covering relation, elements of $D$ may be identified with finite subsets of $N$ covering $N$. Clauses (i), (ii) now read as: (i) more $d \in D$ is larger, more it covers elements of $N$; (ii) given any $x \in N$, we may effectively find some $d=\xi(x) \in D$ covering $x$. We will abbreviate $x \in \operatorname{Cov}(d)$ or " $d$ covers $x$ " by $x \triangleleft d$.

Usually, we take as $\xi(x)$ the smallest $d \in D$ covering $\mathrm{x}$. If $D=N$, we choose $\operatorname{Cov}(d)=[0, d]$, and $\xi(x)=x$ : in this case, $x \triangleleft d$ iff $x \leq d$. If $D=\mathcal{P}_{\text {fin }}(N)$, we choose $\operatorname{Cov}(d)=d$, and $\xi(x)=\{x\}$ : in this case, $x \triangleleft d$ iff $x \in d$.

In applications, we sometimes assume an infinite effective family $\left\{\left(\operatorname{Cov}_{n}, \xi_{n}\right) \mid n \in\right.$ $N$ \} of covering structures to cover $N$ by a fixed $D$ (see section 5 for an example). We read " $x \triangleleft_{l} d$ " as "the $l^{\text {th }}$ component of $d$ covers $x$ ". If we have a family of covering relations, any $d \in D$ may be identified with a record, whose components are finite (and possibly overlapping) subsets $\left\{x \in N \mid x \triangleleft_{l} d\right\}$ of $N$, each of them labelled by some $l \in N$. Plurality of ways of covering $\mathrm{N}$ are introduced only to improve efficiency of the interpretation. Theoretically, the reader may always think that there is just one covering, that all covering are equal to this one, and the index $l$ in $\triangleleft_{l}, \xi_{l}$ is dummy. In fact, the reader may even assume that $D=\mathcal{P}_{\text {fin }}(N)$, and that each $d \in D$ is a finite subset of $\mathrm{N}$.

Cofinality and stationarity on $D$. A subset $X \subseteq D$ is cofinal in $D$ iff for all $d \in D$ there is some $e \in X$ s.t. $d \leq e$. Any cofinal subset is inhabited (there is at least one inhabitant of $D$, and therefore one inhabitant of $X$ above it). A subset $X \subseteq D$ is stationary in $D$ iff for some $e_{0} \in X$ and all $e \geq e_{0}$ we have $e \in X$. Every stationary subset of $D$ is cofinal (but not conversely). Proof. Assume for some $e_{0} \in X$ and all $e \geq e_{0}$ we have $e \in X$. Assume $d \in D$ in order to prove $e \in X$ for some $e \geq d$. We choose $e=j\left(d, e_{0}\right) \geq e_{0}, d$ : we deduce $e \in X$ from $e \geq e_{0}$.

Sequences over $D$, successions. We call any total recursive map $f$ : $D \rightarrow N$ a sequence: we think of $\mathrm{f}$ as a tree of streams of integers, $f\left(d_{0}\right), f\left(d_{1}\right), f\left(d_{2}\right), f\left(d_{3}\right), \ldots$ one for each increasing succession of computation states: $d_{0}<d_{1}<d_{2}<d_{3}<\ldots$ For instance, when $D=N$, the succession $0,12,3, \ldots$ of computation states in $D$ produces the stream of integers: $f(0), f(1), f(2), f(3), \ldots$ We call any total recursive map $g: N \rightarrow I$ (with the elements of $I$ coded in $N$ ) a succession (over $I$ ).

Stationarity and limits. Fix any sequence $f: D \rightarrow N$. We say that $f$ is stationary with limit $n \in N$ iff the set $\{d \in D \mid f(d)=n\}$ is stationary (iff for some $d \in D$ and all $e \geq d$ we have $f(d)=n$ ). We say that $f$ is stationary iff $f$ is stationary with some limit $n \in N$. By directness of $D$, the limit of $f$, when it exists, is unique: if we have both $f(e)=n$ for all $e \geq d$, and $f(e)=m$ for all $e \geq d^{\prime}$, then there is some $d " \geq d, d^{\prime}$, and we 
conclude $m=f\left(d^{\prime \prime}\right)=n$. A key example, in the case $D=N$. Fix any total recursive $f: N \rightarrow \mathcal{B}$. There is a sequence $L=\left\{L_{n}\right\}_{n} \in N$, whose limit is tt when $f(x)=\mathrm{tt}$ for some $x$, and $\mathrm{ff}$ if $f(x)=\mathrm{ff}$ for all $x$. Just choose $L_{n}=f(0) \vee f(1) \vee \ldots \vee f(n) \in \mathcal{B} .\left\{L_{n}\right\}_{n} \in N$ is a stream of ff's, turning to a stream of tt's from the first $x$ such that $f(x)=$ tt (if any exists).

The key point to remark, in order to understand the rest of the paper, is that the limit of $L$ cannot be computed out of $f$. The limit of $L$ is indeed tt or ff, according if $f(x)=\mathrm{tt}$ for some $x \in N$, or $f(x)=\mathrm{ff}$ for all $x \in N$, but in order to decide which is the case, we need Excluded Middle, or the map, non-recursive in $f$, deciding the statement $\exists x .(f(x)=\mathrm{tt})$. In both cases, existence of limit of $\mathrm{L}$ is not intuitionistically proved. Thus, if we want an intuitionistic theory of convergence encompassing $L$, we have to weaken (from an intuitionistic viewpoint) the notion of convergence. The new notion we will use is: $L$ converges iff $L$ changes its value only "finitely many times". This idea will be expressed by Wellfoundation of a suitable "change ordering" on the stream of values of $L$. From a classical viewpoint, this new notion, which we call "stability", is quite close to the traditional one, and equivalent to it when $D=N$ (we skip the proof because it is classical and of no interest here). From a constructive viewpoint, however, "stability" will make the difference. By defining convergence as "stability", we will be able to prove convergence of limits with a non-recursive result. We introduce now intuitionistic Well-foundation, then stability.

Well-foundation (intuitionistic definition). Let $(I, R)$ be any pair of a set $I$ and some binary relation $R \subseteq I \times I$. We define the notion " $(I, R)$ is well-founded". If $P \subseteq I$, we consider $P$ as a property defined over $I$, and we write $P(z)$ for $z \in P$. We say that a property $P$ is $(I, R)$-inductive iff for all $x \in I$ : if $P(y)$ for all $y \in I$ such that $R(y, x)$, then $P(x)$. We say that $(I, R)$ is well-founded on $x \in I$ iff for all $P \subseteq I$ which are $(I, R)$-inductive we have $P(x)$. We say $(I, R)$ is well-founded iff $(I, R)$ is well-founded on all $x \in I$; equivalently, iff for all $P \subseteq I$ which are $(I, R)$-inductive we have $P=I$. Some examples: $(N,<)$ is well-founded. $(Z,<)$, with $Z=$ \{relative integers $\}$, is not well-founded, because the empty set is inductive on $Z$, but $\emptyset \neq Z$. Being well-founded is a proof principle: if $(I, R)$ is well-founded and $P \subseteq I$ is $(I, R)$-inductive, then in order to prove $P=I$ it is enough to prove that $P$ is $(I, R)$-inductive. " $I$ is $R$-well-founded on $x "$ is itself an inductive property in the variable $x$. When no ambiguity will arise, we will drop any reference to $I$, or to $R$, or to both. In this case we will say: " $P$ is inductive", " $x \in I$ is well-founded", " $I$ is well-founded", " $R$ is well-founded".

If $x \in I$ is well-founded, and $R$ is recursive, then for all infinite successions $x_{0}, x_{1}, x_{2}, \ldots, x_{n}, \ldots \in I$, with $x_{0}=x$, we have $R\left(x_{n}+1, x_{n}\right)$ false for some $n \in N$ (proof: by induction over $x$ ). Classically, we may prove for any $R$ something more, namely that " $x$ is well-founded" is equivalent to the fact that all $R$-decreasing chain from $x$ are finite. Intuitionistically, by induction over $n \in N$, we may prove only a weak form of converse: if all $R$-decreasing chain from $x$ have length $\leq n$, then $x$ is well-founded. There are well-founded $x$ with no common bound $n \in N$ to the length of $R$ decreasing sequences from $x$. For instance, add an element $\omega$ to $N$, with 
the axioms $n<\omega$ for all $n \in N$. Then $\omega$ is well-founded in $(N \cup\{\omega\},<)$, but no $n \in N$ is a bound to the length of decreasing sequences from $\omega$. Indeed,for all $n$ there are decreasing sequences of length $>n$ from $\omega$ : $\omega>n>n-1>n-2>\ldots>1>0$

Lexicographic ordering. Let $L \subseteq N$ be any set of integer codes of lists, ordered by list extension. We define the lexicographic ordering over $L$ by: $<x_{0}, \ldots, x_{n-1}>><y_{0}, \ldots, y_{m-1}>$ iff either the first list is an extension of the second one, or for some $i<n, m$ we have $x_{0}=y_{0}, \ldots, x_{i-1}=y_{i-1}$, and $x_{i}>y_{i}$ (in the integer ordering). We quote now two properties of well-founded sets which will be useful later: (1) if $I, J$ are ordering and $f: I \rightarrow J$ is any strictly increasing map, then $J$ well-founded implies $I$ well-founded. (2) If a set $L$ of lists is well-founded by extension ordering, then $L$ is well-founded by lexicographic ordering, too.

\section{$2.1 \quad$ Stability}

Ershov's Change ordering. Stability will express the convergence for a sequence $f$. Intuitively, $f$ is a tree of streams of integers, and stability will say that, in all such streams, the value of $f$ cannot keep on changing forever (eventually, it "stabilizes"). We say that f "changes its mind" between $d \leq e \in D$ iff there are $d \leq d^{\prime} \leq e^{\prime} \leq e$ such that $f\left(d^{\prime}\right) \neq f\left(e^{\prime}\right)$. In this case we write $d<_{f} e$. The relation $<_{f}$ is a sub-ordering of the (strict) ordering on $D$, and $d \leq d^{\prime}<_{f} e^{\prime} \leq e$ implies $d<_{f} e$. We call $<_{f}$ the change ordering (first considered, but for a quite different purpose, by Ershov (see [0])).

Definition 1. (Stability). We say that $\mathrm{f}$ is stable iff $>_{f}$ (not $<_{f}$, but its dual, $>_{f}$ ) is well-founded.

Classically, $f$ is stable iff on all increasing succession $\left\{a_{n}\right\}_{n \in N}$ on $D$, $f$ changes its value only finitely many times. We interpreted increasing succession on $D$ as history of some computations: thus, intuitively speaking, $f$ is stable iff $f$ is stationary when restricted to the history of any computation. We skip the proof of this equivalence because it is classical, and of no interest here. Intuitively, this means that we consider $f$ a convergent sequence iff the succession $\left\{f\left(d_{i}\right)\right\}_{i \in N}$ is convergent for all $d_{0}<d_{1}<d_{2}<d_{3}<\ldots$ (for all computations described by some increasing succession over $D$ ). As we anticipated in the introduction, there is no room, in this simplified picture, for limits which are convergent respect to some computation and divergent respect to some other computation. "f stable" is an intuitionistic proof principle. Let $P \subseteq D$, and assume $P$ is $\left(D,>_{f}\right)$-inductive. That is, assume, for all $d \in D$ : if $P(e)$ for all $e>_{f} d$, then $P(d)$. Then we may conclude that $P=D$.

A (crucial) example of stability. The sequence $L=\left\{L_{n}\right\}_{n \in N}$ defined above is not intuitionistically stationary, yet it is intuitionistically stable. $L$ may change its value at most once, from some $\mathrm{n}$ such that $L_{n}=1$, to some $m>n$ such that $L_{m}=0$. Thus, all $>_{L}$-decreasing chains have length $\leq 1$. This implies that $\left(D,>_{L}\right)$ is well-founded. Thus, stability 
expresses convergence of $L$, even if cannot decide whether $L$ has limit 0 or 1 . More in general, if there is a bound $\mathrm{n}$ to the length of $>_{L}$-decreasing chains, then $>_{L}$ is well-founded and $L$ is stable. This is a particular case of stability: usually, there is no bound $n \in N$ to the length of $>_{L^{-}}$ decreasing chains, or at least no recursive way to compute this bound out of $L$.

Another example of stability. In the case $D=N$, a sequence $f: D \rightarrow N$ is a single stream of values: $a, a, a, a, b, b, b, c, c, c, c, d, d, d, e, e, e, e, e, \ldots \ldots$. Split the stream in adjacent segments each time $f$ changes its value: we get a succession of segments $a, a, a, a, b, b, b, \quad c, c, c, c, \quad d, d, d, \quad e, e, e, e, e, \ldots$ We have $i>_{f} j$ iff walking from $i$ to $j$ we change segment. $f$ stable means $>_{f}$ well-founded, that is, that the segments above are well-founded by $>$. This is an indirect way to say that there are only finitely many such segments (finitely many values of $f$ ), and, in particular, that there is a last segment (corresponding to the last and limit value of $f$ ). By expressing this fact in term of Well-foundation, we do not have to precise how many values $f$ has, nor which is the last one (the limit of $f$ ). This weakening of the notion of convergence (weakening which is such only intuitionistically) will allow us to prove convergence (in an intuitionistic way) also for limits with a non-recursive result.

\section{A limit completion $N^{*}$ of $N$}

In this section we define the set $N^{*}$ of converging sequences over $N$, and the notion of effective maps over $N^{*}$, then we check that our definitions are well-given.

Definition 2. (one-step completion $N^{*}$ of $N$ ). We call one-step completion of $N$ w.r.t. $D$, or just " completion" for short, the set: $N^{*, D}=\{$ stable (total, recursive) sequences $f: D \rightarrow N\}$. For all $I \subseteq N$, we call $I^{*, D}$ the set of all stable (total, recursive) sequences $f: D \rightarrow I$.

We will drop the superscript $D$, and write $I^{*}$ for $I^{*, D}$ whenever no confusion arises. We code the elements of $N^{*}$ by the integer codes of the stable maps $f$. Each integer $n \in N$ is identified with the sequence $n^{\circ, D} \in N^{*}$, constantly equal to $n: n^{\circ, D}(d)=n$ for all $d \in D$. When no confusion arises, we will drop the superscript $D$, and write $n^{\circ}$, or simply $n$. Classically, all stable sequences have a (unique) limit, which cannot be computed uniformly on $N^{*}$ : the existence of such a limit is not an intuitionistic theorem (see the example of the family of stable sequences $L$ in the previous section). Call an isomorphism between $N, N^{*}$ any bijection compatible with zero and successor. Classically, $N$ and $N^{*}$ have the same elements, but there is no recursive isomorphism between them. Otherwise, the image of any $L \in N^{*}$ under this bijection would be the limit value of $L$, and this limit would computable from $L$, contradicting the fact that limit is not computable. From an effective viewpoint, $N^{*}$ and $N$ have a quite different structures: as we said, computable maps 
over $N^{*}$ correspond, via identification of a limit with its own result, to a class of non-recursive maps (actually, to $\Delta_{2}^{0}$-maps).

Fix any two element subset $\mathcal{B}=\{\mathrm{tt}, \mathrm{ff}\}$ of $N$. We call any $b \in \mathcal{B}^{*}$ a stable Boolean. We continue our model construction by defining (by a suitable equation) effective continuous maps over $N^{*}$. Then we define (by topological adherence) the predicate " $b$ is equal to true" for any stable boolean $b$. Eventually, we define equality over $N^{*}$. If $\underline{x}=x_{1}, \ldots, x_{k}$ is any vector of elements of $N^{*}$, let us we abbreviate $x_{1}(d) \ldots, x_{k}(d)$ by $\underline{x}(d)$.

Lifting. For each (total, recursive) map $g: N^{k} \rightarrow N^{*}$ ( $k$-ary) we define its lifting, or extension, to $\left(N^{*}\right)^{k}$. Lifting is a (total, recursive) map $g^{*, D}:\left(N^{*}\right)^{k} \rightarrow N^{*}$, defined by: $g^{*, D}(\underline{x})(d)=g(\underline{x}(d))(d) \in N$, for all $\underline{x} \in\left(N^{*}\right)^{k}$ and $d \in D$. We call $g^{*, D}$ the lifting of $g$. Our definition makes sense: we have $\underline{x}(d) \in N^{k}$, therefore $g(\underline{x}(d)) \in N^{*}$, thus $g(\underline{x}(d))(d) \in N$. We will have to prove that $g^{*, D}:\left(N^{*}\right)^{k} \rightarrow N^{*}$. When no confusion arise, we will drop the index $D$, and write $g^{*}$ for $g^{*, D}$.

Continuous maps. We say that a (total, recursive) $\operatorname{map} \phi:\left(N^{*}\right)^{k} \rightarrow N^{*}$ is continuous iff $\phi$ is extensionally equal to some lifting (to $f^{*}$, for some $f: N^{k} \rightarrow N^{*}$ ). Remark that $\phi$ is determined by its restriction $f$ to $N^{k}$. From a topological viewpoint, this means that $N$ is dense within its completion $N^{*}$.

Extending recursive maps to continuous maps. Every total recursive $h$ : $N^{k} \rightarrow N$ may be extended to some continuous map $h^{\circ, D}$. We define $h^{\circ, D}(\underline{x})(d)=h(\underline{x}(d))$, for all $\underline{x} \in\left(N^{*}\right)^{k}$ and $d \in D$. Again, we will drop the index $D$ if no confusion arises. $h^{\circ}$ extends $h$ to $N^{*}$, in the sense that for all $\underline{x} \in\left(N^{*}\right)^{k}$ we have $h^{\circ}\left(\underline{x}^{\circ}\right)=h(\underline{x})^{\circ}$. Proof. By unfolding definitions, we get $h^{\circ}\left(\underline{x}^{\circ}\right)(d)=h\left(\underline{x}^{\circ}(d)\right)=h(\underline{x})=h(\underline{x})^{\circ}(d)$ for all $\underline{x} \in\left(N^{*}\right)^{k}$ and $d \in D . h$ is continuous. Proof. Define $h^{\prime}: N^{k} \rightarrow N^{*}$ by $h^{\prime}(\underline{x})=h(\underline{x})^{\circ}$ for all $\underline{x} \in N^{k}$. Then $h^{\circ}=\left(h^{\prime}\right)^{*}$, therefore $h$ is continuous. Indeed, by unfolding definition, we get, for all $\underline{x} \in\left(N^{*}\right)^{k}$ and $d \in D: h^{\circ}(\underline{x})(d)=$ $h(\underline{x}(d))=h(\underline{x}(d))^{\circ}(d)=h^{\prime}(\underline{x}(d))(d)=\left(h^{\prime}\right)^{*}(\underline{x})(d)$. If we think of $N$ as a subset of $N^{*}$, we may think of "०" as a special case of "*".

Some examples of extension of a total recursive map to some continuous map. If $x, y \in N^{*}$, then $\left(x+{ }^{\circ} y\right) \in N^{*}$ is defined pointwise, by $\left(x+{ }^{\circ}\right.$ $y)(d)=(x(d)+y(d))$ for all $d \in D$. Another example. Let if $: \mathcal{B}, N, N \rightarrow$ $N$ be a map such that if $(a, b, c)=b$ when $a=\mathrm{tt}$, if $(a, b, c)=c$ when $a=\mathrm{ff}$, for all $b, c \in N$. Take $D=N$, and assume $l \in \mathcal{B}^{*}$ is any (stable) flow of tt's and ff's: tt, ff, ff, tt, tt, tt, ff, tt, ... Then if ${ }^{\circ}\left(l, b^{\circ}, c^{\circ}\right)$ is (for all $b, c \in N$ ) a (stable) flow of $b$ 's and $c$ 's: $b, c, c, b, b, b, c, b, \ldots$. Remark that if $^{\circ}$ does not decide whether $l$ is cofinally 0 or cofinally 1 (in fact, there is no way to make such decision, in general).

Truth over $\mathcal{B}^{*}$. Let $b \in \mathcal{B}^{*}$ a stable boolean. We say that " $b$ is equal to tt", or that " $b$ is tt", for short, iff the set $\{d \in D \mid b(d)=t \mathrm{t}\}$ is cofinal in $D$. From a topological viewpoint, when $D=N$, " $b$ is tt" means that the integer $t \mathrm{t}$ is adherent to the range $\{b(0), b(1), b(2), \ldots\}$ of the sequence $b: N \rightarrow \mathcal{B}$. Classically, if $b \in \mathcal{B}^{*}$ then $b$ is true iff $b$ has limit tt. If $b \in \mathcal{B}^{*}$, then a sufficient condition for $b$ true is that $b$ has limit tt: i.e., that $b(e)=\mathrm{tt}$ for some $e_{0} \in D$ and all $e \geq e_{0}$. Proof: if $\{d \in D \mid b(d)=\mathrm{tt}\}$ is stationary in $D$, then it is cofinal in $D$. All constants and operations over $\mathcal{B}:$ tt $, \mathrm{ff}, \neg, \wedge, \vee, \Rightarrow$ may be extended to constants and operations over 
$\mathcal{B}^{*}: \mathrm{tt}^{\circ}, \mathrm{ff}^{\circ}, \neg^{\circ}, \wedge^{\circ}, \vee^{\circ}, \Rightarrow^{\circ}$. For instance, if $b, c \in \mathcal{B}^{*}$, then $\left(b \Rightarrow^{\circ} c\right) \in \mathcal{B}^{*}$ is defined by: $\left(b \Rightarrow^{\circ} c\right)(d)=(b(d) \Rightarrow c(d)) \in \mathcal{B}$, for all $d \in D$.

Properties over $N^{*}$. Fix any continuous map $P^{*}: N^{k} \rightarrow \mathcal{B}^{*}$. We may consider $P^{*}$ a property over $\left(N^{*}\right)^{k}$ by defining, for all $\underline{x} \in\left(N^{*}\right)^{k}: \underline{x}$ satisfies $P^{*}$ iff $P^{*}(\underline{x})$ is true (as element of $\mathcal{B}^{*}$ ). We call "property over $N^{*}$ " any $P^{*}$ as above. Any decidable property $Q: N^{k} \rightarrow \mathcal{B}$ may be raised, first, to a continuous map $Q^{\circ}:\left(N^{*}\right)^{k} \rightarrow \mathcal{B}^{*}$, second, to a property over $\left(N^{*}\right)^{k}$, which we still denote by $Q^{\circ} . Q^{\circ}$ is an extension of $Q$. Proof. By unfolding definitions, for all $\underline{x} \in N^{k}, Q^{\circ}\left(\underline{x}^{\circ}\right)$ is equivalent to $Q^{\circ}\left(\underline{x}^{\circ}\right)(d)=$ tt cofinally in $D$. We have $\bar{Q}^{\circ}\left(\underline{x}^{\circ}\right)(d)=Q\left(\underline{x}^{\circ}(d)\right)=Q(x)$ for all $d \in D$ : we conclude that $Q^{\circ}\left(\underline{x}^{\circ}\right)(d)=$ tt cofinally in $\mathrm{D}$ iff $Q(x)=\mathrm{tt}$.

Equality over $N^{*}$. Let $={ }^{\circ, D}$ be the lifting of the characteristic map for the equality. We say that $l, m \in N^{*}$ are equal iff $\left(l={ }^{\circ, D} m\right)$ is true, as element of $\mathcal{B}^{*}$. Again, we drop the index $D$ whenever no confusion arises. For any $l, m \in N^{*}$, the element $\left(l={ }^{\circ} m\right) \in \mathcal{B}^{*}$ is a sequence, defined as follows: for all $d \in D$, we have $\left(l={ }^{\circ} m\right)(d)=(l(d)=m(d))=$ tt if $l(d), m(d)$ are equal integer, and $\left(l={ }^{\circ} m\right)(d)=$ ff otherwise. Thus, $l, m \in N^{*}$ are equal iff $\{d \in D \mid(l(d)=m(d)) i n N\}$ is cofinal in $D$ (iff equality of $l(d), m(d)$ holds cofinally). Classically, $l, m$ are equal iff $l, m$ have the same limit. Hence the equality over $N^{*}$ is not decidable.

This completes our model construction.

\subsection{Correctness of the Limit construction}

We have still to check that equality, projections, compositions of continuous and lifting are well-defined. Then we will check that continuous maps are exactly $\Delta_{2}^{0}$-maps, and, in particular, that they include all decision maps for one-quantifier formulas. We need one more concept: pointwise truth and pointwise implication.

Pointwise truth and implication. Let $a, b \in \mathcal{B}^{*}$. We say that a is pointwise true iff $a(d)=$ tt for all $d \in D$. If a is true pointwise, then with more reason a is true cofinally, that is, true as element of $\mathcal{B}^{*}$. We say that $a$ implies $b$ pointwise iff $a(d)=$ tt implies $b(d)=\mathrm{tt}$, for all $d \in D$ (iff $a \Rightarrow^{\circ} b$ is true pointwise). We start by proving a technical result, that the set of true stable booleans is a (proper) filter, w.r.t. the pointwise implication.

Lemma 3. (Filter Lemma). Let $F=\left\{b \in \mathcal{B}^{*} \mid b\right.$ is true $\}$. Then $F$ is a proper filter, w.r.t. the pointwise implication. By this we mean: for all $a, b \in \mathcal{B}^{*}$

1. If $a, b$ are true, then $\left(a \wedge^{\circ} b\right)$ is true.

2. If $a$ is true, and a implies $b$ pointwise, then $b$ is true.

3. $\mathrm{tt}^{\circ}$ is true (pointwise). $\mathrm{ff}^{\circ}$ is not true.

\section{Proof}

1. Fix any $d \in D$. We have to prove that there is some $e \geq d$ s.t. $a(e)=b(e)=$ tt. We argue by induction over $d \in D$, w.r.t. order $>_{a}$, well-founded because $a \in \mathcal{B}^{*}$. By the assumption " $a$ is true", 
there is some $e \geq d$ such that $a(e)=$ tt. By the assumption " $b$ is true", there is some $e^{\prime} \geq e \geq d$ such that $b\left(e^{\prime}\right)=\mathrm{tt}$. If $a\left(e^{\prime}\right)=a(e)$, then $a\left(e^{\prime}\right)=b\left(e^{\prime}\right)=\mathrm{tt}$. Now assume $a\left(e^{\prime}\right) \neq a(e)$. Since $e^{\prime} \geq e \geq d$, then $e^{\prime}>_{a} d$, and by inductive hypothesis over $e^{\prime}$, we conclude that there is some $e^{\prime \prime} \geq e^{\prime} \geq d$ such that $a\left(e^{\prime \prime}\right)=b\left(e^{\prime \prime}\right)=$ tt.

2. For all $d$ there is some $e \geq d$ s.t. $a(e)=$ tt. Then $b(e)=$ tt by pointwise implication. Thus, $b(e)=$ tt holds cofinally in $D$.

3. $\operatorname{tt}^{\circ}(d)$ is always equal to $t t$, therefore it is pointwise true. $f f^{\circ}(d)$ is always equal to $\mathrm{ff}$, therefore it is not cofinally true.

Point 1 of lemma 3 says that the intersection of two particular cofinal subsets of $D$ (the subset of $d \in D$ such that $a(d)=\mathrm{tt}$, and of $d \in D$ such that $b(d)=\mathrm{tt})$ is still cofinal in $D$. This is far from being true for generic cofinal subsets of $D$ (think of even and odd integers in $D=N$ ). In this particular case, however, the intersection is still cofinal, thanks to stability conditions for $a, b \in \mathcal{B}^{*}$. Tautological consequences. Filter Lemma 3 implies that the set of true propositions defined using $\neg^{\circ}, \wedge^{\circ}, \vee^{\circ}, \Rightarrow^{\circ}$ over elements of $\mathcal{B}^{*}$ are closed under tautological consequences. Proof. Assume $\alpha_{1}, \ldots, \alpha_{n}$ are true propositions, and $\alpha$ is a tautological consequence of $\alpha_{1}, \ldots, \alpha_{n}$. Then $\alpha_{1} \wedge^{\circ} \ldots \wedge^{\circ} \alpha_{n}$ is true by Filter Lemma 3, and $\left(\alpha_{1}, \ldots, \alpha_{n} \Rightarrow^{\circ} \alpha\right)$ is true pointwise because it is a tautology. Again by the Filter Lemma, we conclude that $\alpha$ is true.

Out of the Filter Lemma we may start checking some correctness conditions for our model. Let $<_{-},{ }_{-}>: N, N \rightarrow N$ be any coding of pairs in $N$.

Lemma 4. (Correctness). (i) Any n-tuple of stable sequences is a stable sequence. (ii) Equality on stable sequences is an equivalence relation. (iii) Continuous maps include constant maps, projections, and are closed under compositions. (iv) Lifting produces maps compatible with equality on stable sequences

Proof. (i) Take $n=2$ (this case implies the general case). Let $a, b: D \rightarrow$ $N$ stable, and $c=<a, b>^{\circ}$ denote the map $d \mapsto<a(d), b(d)>$. We have to prove that $D$ is well-founded w.r.t. $>_{c}$, that is, that any $d \in D$ is wellfounded w.r.t. $>_{c}$. We will prove a little more, namely that for all $d_{1}, d_{2} \in$ $D$, and all $d \geq d_{1}, d_{2}$, we have $d$ well-founded by $>_{c}$. The thesis will follows by taking any $d_{1}=d_{2}=d \in D$. We argue by principal induction over $d_{1},>_{a}$ and secondary induction over $d_{2},>_{b}$. Well-foundation w.r.t. $>_{c}$ is inductive: thus, it is enough to check that for all $e>_{c} d$, we have $e$ well-founded. By definition of $>_{c}$, for some $d^{\prime}, e^{\prime}$ such that $d \leq d^{\prime}<e^{\prime} \leq$ $e$, we have $<a\left(d^{\prime}\right), b\left(d^{\prime}\right)>\neq<a\left(e^{\prime}\right), b\left(e^{\prime}\right)>$, that is, either $a\left(d^{\prime}\right) \neq a\left(e^{\prime}\right)$ or $b\left(d^{\prime}\right) \neq b\left(e^{\prime}\right)$. This means either $\left(d_{1} \leq d<_{a} e\right)$ or $\left(d_{2} \leq d<_{b} e\right)$. In the first case we apply principal induction hypothesis over the pair: $e, e$. In the second case we apply secondary induction hypothesis over the pair: $d_{1}, e$. (ii) Fix any three stable sequences $a, b, c: D \rightarrow N$.

Reflexivity. $a={ }^{\circ} a$ is pointwise true.

Symmetry. Assume $a={ }^{\circ} b$ is true. The implication $\left(a={ }^{\circ} b \Rightarrow^{\circ} b={ }^{\circ} a\right)$ is true pointwise: for all $d \in D, a(d)=b(d)$ implies $b(d)=a(d)$. By 3 , point 2 , also $b={ }^{\circ} a$ is true. 
Transitivity. Assume $a={ }^{\circ} b$ and $b={ }^{\circ} c$ are true. Then by 3, point 1 , $\left(a={ }^{\circ} b \wedge b={ }^{\circ} c\right)$ is true. The implication $\left(a={ }^{\circ} b \wedge b={ }^{\circ} c \Rightarrow{ }^{\circ} a={ }^{\circ} c\right)$ is pointwise true: for all $d \in D, a\left(d^{\prime}\right)=b\left(d^{\prime}\right) \wedge b\left(d^{\prime}\right)=c\left(d^{\prime}\right)$ implies $a\left(d^{\prime}\right)=c\left(d^{\prime}\right)$. By 3, point 2, we conclude that $a={ }^{\circ} c$ is true.

(iii) Constant maps. If $f(\underline{x})(d)$ is constantly $n$, then $f^{*}$ is constantly $n^{\circ}: f^{*}(\underline{x})(d)=f(\underline{x}(d))(d)=n=n^{\circ}(d)$. Thus, constant maps are continuous. Projections. Let $q_{i}$ be the $i$-th projection over $N^{k}$, and $p_{i}$ the $i$-th projection over $\left(N^{*}\right)^{k}$. We check that $p_{i}, q_{i}^{\circ}$ are extensionally equal. Indeed, $q_{i}^{\circ}(\underline{x})(d)=q_{i}(\underline{x}(d))=x_{i}(d)=p_{i}(\underline{x})(d)$ for all $\underline{x} \in\left(N^{*}\right)^{k}, d \in D$. Thus, $p_{i}$ is continuous. Compositions. Let $g$ be a map of arity $n$, and $\underline{h}$ be any vector of $n$ maps of arity $m$. Then $g \circ h$ is defined $: N^{m} \rightarrow N$. We check that $\left(g^{*} \circ \underline{h}^{*}\right)(\underline{x})=\left(g^{*} \circ \underline{h}\right)^{*}(\underline{x})$ for all $\underline{x} \in\left(N^{*}\right)^{k}$. By definition unfolding, for all $d \in D$ we have: $g^{*}\left(\underline{h}^{*}(\underline{x})\right)(d)=g\left(\underline{h}^{*}(\underline{x})(d)\right)(d)=$ $g(\underline{h}(\underline{x}(d))(d))(d)=g^{*}(\underline{h}(\underline{x}(d)))(d)=\left(g^{*} \circ \underline{h}\right)(\underline{x}(d))(d)=\left(g^{*} \circ \underline{h}\right)^{*}(\underline{x})(d)$. We conclude that $\left(g^{*} \circ \underline{h}^{*}\right)$ is the lifting of $\left(g^{*} \circ \underline{h}\right)$. Thus, $\left(g^{*} \circ \underline{h}^{*}\right)$ is continuous.

(iv) Assume $f: N^{k} \rightarrow N^{*}$, in order to show $f^{*}:\left(N^{*}\right)^{k} \rightarrow N^{*}$. Let $\underline{x} \in\left(N^{*}\right)^{k}$ : we have to check that the sequence $f^{*}(\underline{x})():. D \rightarrow N^{k}$ is stable, or that $>_{f}(\underline{x})$ is well-founded. We will prove some Claim slightly more general than our thesis: (Claim) for any three $d \geq d_{1}, d_{2} \in D, d$ is well-founded under $>_{f(\underline{x})}$. Eventually, our thesis, or "all $d \in D$ are wellfounded under $>_{f(\underline{x})}$ ", will follow from the Claim, just by taking $d_{1}=$ $d_{2}=d \in D$. By point (i) of this Lemma, $\underline{x}():. D \rightarrow N^{k}$ is stable, and $>_{\underline{x}}$ is well-founded. Thus, we may prove the Claim by principal induction over $d_{1},>_{\underline{x}}$, and secondary induction over $d_{2},>_{f(\underline{i})}$, with $\underline{i}=\underline{x}\left(d_{1}\right)$. Wellfoundation of $d \in D$ is an inductive property: thus, it is enough to prove that all $e \in D$ with $e>_{f(\underline{x})} d$ are well-founded under $>_{f(\underline{x})}$. By definition of $e>_{f(\underline{x})} d$, and of $f^{*}$, for some $d^{\prime}, e^{\prime}$ such that $d_{1}, d_{2} \leq d \leq d^{\prime}<e^{\prime} \leq e$ we have $f(\underline{x})\left(d^{\prime}\right)=f\left(\underline{x}\left(d^{\prime}\right)\right)\left(d^{\prime}\right) \neq f\left(\underline{x}\left(e^{\prime}\right)\right)\left(e^{\prime}\right)=f(\underline{x})\left(e^{\prime}\right)$. There are two subcases.

Assume either $\underline{x}\left(d_{1}\right) \neq \underline{x}\left(d^{\prime}\right)$, or $\underline{x}\left(d^{\prime}\right) \neq \underline{x}\left(e^{\prime}\right)$. Then $\left(d_{1}<_{\underline{x}} e\right)$. By ind. hypothesis over the pair $e, e$, and $e \geq e$, we deduce that $e$ is well-founded under $>_{f}(\underline{x})$.

Assume $\underline{x}\left(d_{1}\right)=\underline{x}\left(d^{\prime}\right)=\underline{x}\left(e^{\prime}\right)$. Call $\underline{i}$ the common value of these three expressions. By hypothesis on $d^{\prime}, e^{\prime}$, we deduce $f(\underline{i})\left(d^{\prime}\right)=f\left(\underline{x}\left(d^{\prime}\right)\right)\left(d^{\prime}\right) \neq$ $f\left(\underline{x}\left(e^{\prime}\right)\right)\left(e^{\prime}\right)=f(\underline{i})\left(e^{\prime}\right)$. Thus, $\left(d_{2}<_{f(i)} e\right)$. By $i=\underline{x}\left(d_{1}\right)$, and ind. hyp. over the pair $d_{1}, e$, and $e \geq d_{1}, e$, we conclude that $e$ is well-founded by $>_{f(\underline{x})}$.

The last lemma to be proved says that any lifting $f^{*}$ only depends on the extensional behavior $\left(\operatorname{modulo}={ }^{\circ}\right.$ ) of the map $f$.

Lemma 5. (Compatibility of ${ }^{*}$ and Extensional Equality). If $f, g: N^{k} \rightarrow$ $N^{*}$ are extensionally equal, then $f^{*}, g^{*}$ are extensionally equal over $\left(N^{*}\right)^{k}$.

Proof. Let $\underline{x} \in\left(N^{*}\right)^{k}$. We have to prove that $f^{*}(\underline{x})={ }^{\circ} g^{*}(\underline{x})$, that is, that for all $d \in D$ there is some $e \geq d$ such that $f(\underline{x}(e))(e)=g(\underline{x}(e))(e)$. We argue by induction over $>_{\underline{x}}$ (well-founded by Lemma 4 , point (i)). 
By assumption, f, g are extensionally equal over $N^{k}$ : for all $\underline{i} \in N$ we have $f(\underline{i})={ }^{\circ} g(\underline{i})$ in $N^{*}$, that is, $\{e \in D \mid f(\underline{i})(e)=g(\underline{i})(e)\}$ is cofinal in $D$. Taking $\underline{i}=\underline{x}(d)$, we deduce that for all $d$, there is some $e \geq d$ such that $f(\underline{x}(d))(e)=g(\underline{x}(d))(e)$. If $\underline{x}(d)=\underline{x}(e)$ we get the thesis. If $\underline{x}(d) \neq \underline{x}(e)$, we have $e>_{x} d$. By induction hypothesis on $e$ there is some $p$ such that $p \geq e \geq d$ and $f(\underline{x}(p))(p)=g(\underline{x}(p))(p)$. We get our thesis also in this case.

Fix any map $f: N \rightarrow N^{*}$, constantly equal, in $N^{*}$, to $n^{\circ}$ : that is, $f(x)={ }^{\circ} n^{\circ}$ for all $x \in N$. Define a map $g: N \rightarrow N^{*}$ by $g(x)=n^{\circ}$ for all $x \in N$. Then $f, g$ are extensionally equal. By $5, f^{*}, g^{*}$ are extensionally equal. By $4, g^{*}$ is constantly $n^{\circ}$. Thus, $f^{*}$ is constantly equal, in $N^{*}$, to $n^{\circ}: f^{*}(L)={ }^{\circ} n^{\circ}$ for all $L \in N^{*}$. The Density Argument. As a particular case, if $P: N \rightarrow \mathcal{B}^{*}$ is an always true property on $N$, then its lifting $P^{*}: N^{*} \rightarrow \mathcal{B}^{*}$ is an always true property on $N^{*}$. Thus, in order to prove $P^{*}(l)$ for all $l \in N^{*}$, it is enough to prove $P(x)$ for all $x \in N$. We call this argument "the Density Argument", because, topologically, and if we think of $P^{*}$ as an open subset of $N^{*}$, it means that $N$ dense in $N^{*}$. By combining the previous Lemmas we conclude Correctness:

Theorem 6. (Correctness of the topological model). All relations and maps used in our model construction are well-defined:

1. (Equality) equality on $N^{*}$ is an equivalence relation;

2. (Category of limits and continuous maps) continuous maps include projections and are closed under composition;

3. (Lifting) the result of lifting is compatible with equality on $N^{*}$; lifting sends extensionally equal maps into extensionally equal maps.

We include here a simple consequence of the Correctness Theorem.

Lemma 7. (Lifting and Completion). Let $X: N \rightarrow \mathcal{B}$ be any recursive subset of $N$. Then we may form both the property $X^{\circ}: N^{*} \rightarrow \mathcal{B}^{*}$ over $N^{*}$, and the subset $X^{*} \subseteq N^{*} . X^{\circ}$ and $X^{*}$ are related as follows: for all $x \in N^{*}, X^{\circ}(x)$ iff $x={ }^{\circ} y$ for some $y \in X^{*}$.

Proof. Assume $X^{\circ}(x)(d)$ is cofinally true. Then $X\left(x\left(d_{0}\right)\right)$ is true for some $d_{0} \in D$, hence $x\left(d_{0}\right) \in X$. Define $y=$ if $^{\circ}\left(X^{\circ}(x), x, x\left(d_{0}\right)^{\circ}\right) \in N^{*}$. We have $y(d) \in X$ for all $d \in D$ because if $X(x(d))=$ tt then $y(d)=$ $x(d) \in X$, while if $X(x(d))=\mathrm{ff}$ then $y(d)=x\left(d_{0}\right) \in X$. Thus, $y \in X^{*}$. We have $x={ }^{\circ} y$ because $X^{\circ}(x)=\mathrm{tt} \Rightarrow^{\circ}$ if $^{\circ}\left(X^{\circ}(x), x, x\left(d_{0}\right)^{\circ}\right)={ }^{\circ} x$ follows by definition of if and Density. Conversely, assume $x={ }^{\circ} y$ for some $y \in X^{*}$. Then $X^{\circ}(x)={ }^{\circ} X^{\circ}(y)={ }^{\circ} t^{\circ}$.

\subsection{The limit construction is independent from D}

In the remaining of the section we will prove, as we anticipated in the introduction, that the construction $N^{*, D}$ does not depend on the direct 
set $D$. For all direct sets $D, E$ covering $N$, we will prove that the structures $N^{*, D}$ and $N^{*, E}$ are recursively isomorphic. By this we mean: there are recursive bijections between learning objects in $N^{*}, D$ and in $N^{*}, E$, and between continuous maps over $N^{*, D}$ and over $N^{*}, E$; and these bijections are compatible with equality, with extensionally equality, and with function application. We need some definitions first. We say that a map $\alpha: D \rightarrow D$ is above the identity iff $\alpha(d) \geq d$ for all $d \in D$. We say that a pair of total recursive weakly increasing maps $\beta: D \rightarrow E, \gamma: E \rightarrow D$ connects two direct sets $D, E$ iff both $\gamma \beta: D \rightarrow D$ and $\beta \gamma: E \rightarrow E$ are above the identity. We say that $D, E$ are connected iff there are $\beta, \gamma$ connecting $D, E$. We will prove that any two countable direct sets $D, E$ covering $N$ are connected, and that if $D, E$ are connected then $N^{*}, D, N^{*}, E$ are recursively isomorphic. The first step is to check:

If $\beta: D \rightarrow E$ is any (total recursive) map, we define a map $g \mapsto g^{\beta}$ from $(E \rightarrow N)$ to $(D \rightarrow N)$ by composition: $g^{\beta}=g \beta$.

Lemma 8. (Weakly increasing maps). Let $D, E$ be recursive direct sets covering $N$. If $\beta: D \rightarrow E$ is weakly increasing, then $g \mapsto g^{\beta}$ is a map from $N^{*, D}$ to $N^{*, E}$.

Proof. Assume $>_{g}$ is well-founded over $D$, in order to prove that $>_{g^{\beta}}$ is well-founded over E. If $e>_{g^{\beta}} e^{\prime}$, then $g \beta\left(e_{1}\right) \neq g \beta\left(e_{2}\right)$ for some $e \geq e_{1}>e_{2} \geq e^{\prime}$. Since $\beta$ is weakly increasing, this implies $g\left(\beta\left(e_{1}\right)\right) \neq$ $g\left(\beta\left(e_{2}\right)\right)$ for some $\beta(e) \geq \beta\left(e_{1}\right)>\beta\left(e_{2}\right) \geq \beta\left(e^{\prime}\right)$. This latter, in turn, implies $\beta(e)>_{g} \beta\left(e^{\prime}\right)$. Thus, $\beta$ is an increasing mapping from $\left(E,>_{g^{\beta}}\right)$ to $\left(D,>_{g}\right)$. From $\left(D,>_{g}\right)$ well-founded we conclude $\left(E,>_{g^{\beta}}\right)$ well-founded.

Lemma 9. (maps above the identity). Assume $D$ is a direct set, $\alpha$ : $D \rightarrow D$ is weakly increasing and above the identity. If $f, g \in N^{*, D}$ and $f={ }^{\circ, D} g$, then $f \alpha, g \alpha \in N^{*, D}, f \alpha={ }^{\circ, D} g \alpha$ and $f \alpha={ }^{\circ, D} f$.

Proof. We have $f \alpha, g \alpha \in N^{*, D}$ by 2.7 , because $\alpha$ is weakly increasing. Now it is enough to check that $f \alpha={ }^{0, D} f$ for all $f \in N^{*, D}$. Then $f \alpha={ }^{\circ, D} g \alpha$ will follows from: $f \alpha={ }^{\circ, D} f, f={ }^{\circ, D} g, g={ }^{\circ, D} g \alpha$, and transitivity of $=^{\circ, D}$. We have to check that for all $d \in D$ there is some $d^{\prime} \geq d$ such that $f \alpha\left(d^{\prime}\right)=f\left(d^{\prime}\right)$. We argue by induction over $d$ and $>_{f}$ . If $f \alpha(d)=f(d)$ we are done. If $f \alpha(d) \neq f(d)$, then from $\alpha(d) \geq d$ we deduce $\alpha(d)>_{f} d$. By induction hypothesis on $\alpha(d)$ we conclude that there is some $d^{\prime} \geq \alpha(d) \geq d$ such that $f \alpha\left(d^{\prime}\right)=f\left(d^{\prime}\right)$.

Lemma 10. (Connection Lemma). Let D, E be recursive direct sets covering $N$. If $\beta, \gamma$ connects $D, E$, then $f \mapsto f^{\gamma}, g \mapsto g^{\beta}$ are maps compatible with ${ }^{\circ}, D,={ }^{\circ, E}$. 
Proof. Assume $f={ }^{\circ, D} f^{\prime}$, in order to prove $f \gamma={ }^{\circ}, E f^{\prime} \gamma$. By 9 , and $\gamma \beta$ above the identity, we deduce $f \gamma \beta={ }^{\circ}, D f^{\prime} \gamma \beta$. This means that the set of $d \in D$ such that $f \gamma \beta(d)=f^{\prime} \gamma \beta(d)$ is cofinal in $D$. Fix now any $e \in E$. Our thesis is: there is some $e^{\prime} \geq e$ such that $f \gamma\left(e^{\prime}\right)=f^{\prime} \gamma\left(e^{\prime}\right)$. By what we said, for some $d^{\prime} \geq g(e)$ we have $f \gamma \beta\left(d^{\prime}\right)=f^{\prime} \gamma \beta\left(d^{\prime}\right)$. By $d^{\prime} \geq \gamma(e)$ and $\beta$ weakly increasing, we deduce $\beta\left(d^{\prime}\right) \geq \beta \gamma(e) \geq$ (by $\beta \gamma$ above the identity) $e$. Thus, $f \gamma\left(\beta\left(d^{\prime}\right)\right)=f^{\prime} \gamma\left(\beta\left(d^{\prime}\right)\right)$ and $\beta\left(d^{\prime}\right) \geq e$. The thesis now follows by choosing $e^{\prime}=\beta\left(d^{\prime}\right) \in E$. Assume $g={ }^{\circ, E} \bar{g}^{\prime}$, in order to prove $g \beta={ }^{\circ}{ }^{E} g^{\prime} \beta$. If $D, E$ are connected, then $E, D$ are connected. By what we just said, from $g={ }^{\circ, E} g^{\prime}$ we conclude $g \beta={ }^{\circ, D} g^{\prime} \beta$.

Lemma 11. (Covering Lemma). Assume $D$ is a recursive direct set covering $N$. Then $N, D$ are connected by some $c: N \rightarrow D, h: D \rightarrow E$.

Proof. By assumption, there is some recursive enumeration $d_{0}, d_{1}, d_{2}, \ldots$ of the elements of $D$, and some covering relation Cov, $\xi$ between $D$ and $N$. We will now define $c, h$ connecting $N, D$. The map $c: N \rightarrow D$. There is some total recursive $c: N \rightarrow D$ strictly increasing, with $\{c(x) \mid x \in N\}$ cofinal in $D$. Define $c(0)=$ any element of $D, c(n+1)=j\left(c(n), d_{n}, \xi(i)\right)$, for $i=$ the first element of $N$ not covered by $c(n) \in D$. $c$ is welldefined because $D$ is direct. $c$ is recursive because the maps $j, d(),. \xi$ are. $\{c(x) \mid x \in N\}$ is cofinal in $D$, because each element of $D$ is equal to some $d_{n}$, and $d_{n} \leq c(n)$ by construction. The map $h: D \rightarrow N$. For any $d \in D$, define $h(d)=$ the first $x \in N$ such that $d \leq c(x)$. There is some $x \in N$ such that $d \leq c(x)$ by cofinality of $\{c(n) \mid n \in N\}$. $h$ is recursive because $c$ is. $c$ is strictly increasing. We have $c(n+1)>c(n)$ because $c(n+1) \geq c(n)$ by definition of $c$, and again by definition, $c(n+1)$ covers $i$, while $c(n)$ does not. $h$ is weakly increasing. If $d \leq e$, then $e \leq c(h(e))$ implies $d \leq c(h(e))$. Therefore $h(d) \leq h(e)$, because $h(d)$ is $\leq$ than all $x \in N$ such that $d \leq c(x)$. $c \circ h$ is above the identity. If $h(d)=x$ then $c(x) \geq d$ by definition of $h$. Thus, $c(h(d)) \geq d$. $h \circ c$ is above the identity. We have $h(c(n))=$ the first $x \in N$ such that $(c(n) \leq c(x))=n$, because $c$ is strictly increasing, and if it were $n<x$ we would deduce $c(n)<c(x)$.

We extend isomorphism to maps in the traditional way. Let $\phi: N^{*, D} \rightarrow$ $N^{*, D}$. We define a map $\phi^{\beta, \gamma}: N^{*, E} \rightarrow N^{*, E}$ by $\phi^{\beta, \gamma}(g)=\phi\left(g^{\beta}\right)^{\gamma}$ for all $g \in N^{*, E}$.

Lemma 12. (Isomorphism Lemma). Let D, E be any two direct sets, connected by $\beta, g$. Then $f \mapsto f \gamma, g \mapsto g \beta$ is an isomorphism pair between $N^{*, D}$ and $N^{*, E}$, commuting with application of maps.

Proof. By Lemma 10, $f \mapsto f \gamma, g \mapsto g \beta$ are map from $N^{*, D}$ to $N^{*, E}$, and conversely, compatible with equality. We have still to prove that $f \mapsto f \gamma, g \mapsto g \beta$ is an isomorphism pair. By $\gamma \beta, \beta \gamma$ above the identity, 
and 9, we conclude $f \gamma \beta={ }^{\circ, D} f$ and $g \beta \gamma={ }^{\circ}, E$. Isomorphism commutes with application. We have to check that, for all $\phi: N^{*, D} \rightarrow N^{*, D}$ and $f \in N^{*, D}$, we have $\phi(f)^{\gamma}={ }^{\circ, E} \phi^{\beta, \gamma}\left(f^{\gamma}\right)$. By unfolding definitions, $\phi^{\beta, \gamma}\left(f^{\gamma}\right)=\phi(f \gamma \beta) \gamma={ }^{\circ, E}$ (by $\left.f \gamma \beta={ }^{\circ, D} f\right) \phi(f)^{\gamma}$. In the same way we may check that for all $\psi: I^{*, E} \rightarrow J^{*, E}$ and $g \in N^{*, E}$, we have $\psi(g)^{\beta}={ }^{\circ, D} \psi^{\gamma, \beta}\left(g^{\beta}\right)$.

Theorem 13. (Isomorphism Theorem). Let D, E be any two direct sets covering $N$. Then $N^{*, D}$ and $N^{*, E}$ are recursively isomorphic.

Proof. By Lemma 12 we deduce that the completions taken w.r.t. $D$ and $N$ are effectively isomorphic, for any $D$ covering $N$. By transitivity of isomorphism, we conclude that the completions taken w.r.t. any direct sets $D, E$ covering $N$ are effectively isomorphic.

\section{Characterizing continuous maps on $\boldsymbol{N}^{*}$}

In this section we check that the set continuous maps over $N^{*}$ coincide with the set of maps recursive w.r.t. the Halting Problem; classically, this means that they are exactly all $\Delta_{2}^{0}$-maps. We also claim that continuous maps over $N^{*}$ may be defined using a simple definition schema, pointwise linear recursion. Eventually, we prove, constructively, a conservativity result: equations $g(x)=0$ (with $g$ recursive) solvable in $N^{*}$ are solvable in $N$, too.

Let $\mathcal{B}=\{$ tt, $\mathrm{ff}\}$ be any two element subset of $N$. Let $f: N^{2} \rightarrow \mathcal{B}$ be any binary recursive property, and $\mathbf{s}_{f}(x), \mathbf{f} \mathbf{s t} f(x)$ be (classically) defined by: $\mathbf{s}_{f}(x)=\mathrm{tt}$ if $f(x, y)=\mathrm{tt}$ for some $y,=\mathrm{ff}$ if $f(x, y)=\mathrm{ff}$ for all $y$. first $_{f}(x)=$ the first $y$ s.t. $f(x, y)=$ tt if any $=0$ if $f(x, y)=\mathrm{ff}$ for all $y$.

The maps are non-recursive in general (they solve the Halting Problem). They may be generalized to $(k+1)$-ary recursive properties $f(\underline{x}, y)$. The maps $\mathbf{s}_{f}$, first f $_{f}$ may be (intuitionistically) defined as continuous maps: $\left(N^{*}\right)^{k} \rightarrow \mathcal{B}^{*}$, provided $D$ covers $N$. Take any $D$ equipped with a covering relation Cov, $x$. In the case we have a family $\left(\operatorname{Cov}_{n}, \xi_{n}\right)$ of covering for $N$, we choose (for a reason we will explain later) the covering with index $m=\langle f, \underline{x}\rangle$ (some name for the map $f(\underline{x},$.$) we are considering).$ We abbreviate $y \in \operatorname{Cov}_{m}(d)$ by $y \triangleleft_{m} d$.

Then we may define, for all $\underline{x} \in N^{k}$ and all $d \in D: \mathbf{s}_{f}(\underline{x})(d)=\mathrm{tt}$ if $f(\underline{x}, y)=$ tt for some $y \triangleleft_{m} d,=$ ff if $f(\underline{x}, y)=$ ff for all $y \triangleleft_{m} d$. fst $_{f}(\underline{x})(d)=$ the first $y \in \operatorname{Cov}_{m}(d)$ s.t. $f(\underline{x}, y)=0$, if $f(\underline{x}, y)=$ tt for some $y \triangleleft_{m} d,=0$ if $f(\underline{x}, y)=\mathrm{ff}$ for all $y \triangleleft_{m} d$. In the same way we define a universal quantification $\operatorname{all}_{f}(\underline{x})$ by $\neg \mathbf{S}_{\neg f}(\underline{x})$. These maps are recursive because we assumed $\operatorname{Cov}_{m}(d)$ be finite and recursive in $m, d$. Fix any $\underline{x} \in N^{k}$, and any increasing succession $d_{1}<d_{2}<d_{2}<\ldots$ of computation states. This succession represents the history of some computation. The succession 
$\mathbf{s}_{f}(\underline{x})\left(d_{1}\right), \mathbf{s}_{f}(\underline{x})\left(d_{2}\right), \mathbf{s}_{f}(\underline{x})\left(d_{2}\right), \ldots$

represents instead, for each step $d_{i}$ of the computation, the truth value $\mathbf{s}_{f}(\underline{x})\left(d_{i}\right)$ we assign to $\exists x \in N . f(\underline{x}, y)=$ tt in the step $d_{i}$. We start by assigning ff to $\exists x \in N . f(\underline{x}, y)=$ tt. We switch to tt if and when we reach some $d_{i} \in D$ which $m$-covers some solution of $f(x, y)=\mathrm{tt}$.

Lemma 14. ("some", "first" maps). (i) $\mathrm{s}_{f}(\underline{x}), \mathrm{fst}_{f}(\underline{x}) \in \mathcal{B}^{*}$.

(ii) for all $x, y \in\left(N^{*}\right)^{k}+1$, the implications: $a . \mathbf{s}_{f}(x)=\mathrm{tt} \Rightarrow^{\circ} f(x$, first $(x))={ }^{\circ}$ tt $b . \mathbf{s}_{f}(x)=\mathrm{ff} \Rightarrow^{\circ} f(x, y)={ }^{\circ}$ ff are true in $\mathcal{B}^{*}$ (are cofinally true).

Proof. We start by remarking that, by monotonicity of covering, if $f(\underline{x}, y)=$ tt for some $y \triangleleft_{m} d$, and $e \geq d$, then $f(\underline{x}, y)=$ tt for some $y \triangleleft_{m} e$. That is, if $\mathbf{s}_{f}(\underline{x})(d)=\mathrm{tt}$, then $\mathbf{s}_{f}(\underline{x})(e)=\mathrm{tt}$ for all $e \geq d$, hence $\mathbf{s}_{f}(\underline{x})(d)=\mathbf{s}_{f}(\underline{x})(e)$. By contraposition, if $\mathbf{s}_{f}(\underline{x})(d) \neq \mathbf{s}_{f}(\underline{x})(e)$ and $e \geq d$, we deduce $\mathbf{s}_{f}(\underline{x})(d)=\mathrm{ff}$. We also deduce $\mathrm{s}_{f}(\underline{x})(e)=\mathrm{tt}$, because $\mathbf{s}_{f}(\underline{x})(e) \neq \mathbf{s}_{f}(\underline{x})(d)=\mathbf{f f}$. Now we $e$ claim that $e>_{\mathbf{s}_{f}(x)} d$ implies $\mathbf{s}_{f}(\underline{x})(d)=\mathrm{ff}$ and $\mathbf{s}_{f}(\underline{x})(e)=\mathrm{tt}$. Indeed, $e>\mathbf{s}_{f}(\underline{x}) d$ unfolds to: $\mathbf{s}_{f}(\underline{x})\left(d^{\prime}\right) \neq \mathbf{s}_{f}(\underline{x})\left(e^{\prime}\right)$ for some $d \leq d^{\prime} \leq e^{\prime} \leq e$. We deduce $\mathbf{s}_{f}(\underline{x})\left(d^{\prime}\right)=\mathrm{ff}, \mathbf{s}_{f}(\underline{x})\left(e^{\prime}\right)=\mathrm{tt}$. By $d \leq d^{\prime}$ and contraposition, we deduce $\mathbf{s}_{f}(\underline{x})(d)=\mathrm{ff}$. By $e^{\prime} \leq e$, we obtain $\mathbf{s}_{f}(\underline{x})(e)=\mathrm{tt}$. Thus, $\mathbf{s}_{f}(\underline{x})$ is an increasing map from $D,>\mathbf{s}_{f}(\underline{x})$ to $\mathcal{B}$, ordered by tt $>\mathrm{ff}$. This latter is a well-founded set: we conclude that $>_{\mathbf{s}_{f}(x)}$ is well-founded. (ii.a) Assume $\mathbf{s}_{f}(x)(d)=\mathrm{tt}$. Then, by def. of $\mathbf{s}_{f}, f\left(x, \mathbf{f} \mathbf{s t}_{f}(x)(d)\right)=$ tt for the same $d$. Thus, implication (a) is pointwise true, hence it is cofinally true. (ii.b) Assume $\mathbf{s}_{f}(x)(d)=\mathbf{f f}$, and fix any $y \in N$. Then, by definition of $\mathbf{s}_{f}$, whenever $y \triangleleft_{m} d$ we have $f(x, y)(d)=\mathbf{f f}$. By definition of Covering, $y \triangleleft_{m} \xi_{m}(y)$. By monotonicity of covering, for all $e \geq \xi_{m}(y)$ we have $y \triangleleft_{m} e$. Therefore: (b) $\mathbf{s}_{f}(x)(e)=\mathrm{ff} \Rightarrow f(x, y)(e)=\mathrm{ff}$ for all $e \geq \xi_{m}(y)$. In particular, (b) is true for $e=j\left(d, \xi_{m}(y)\right) \geq d, \xi_{m}(y)$, for all $d \in D$. Therefore (b) is cofinally true, or true in $\mathcal{B}^{*}$. By a Density argument, (b) is true for all $y \in N^{*}$.

Interpreting Quantifiers. By definition of $\mathbf{s}_{f}(\underline{x})$, the equivalence $(1) \mathbf{s}_{f}(\underline{x})={ }^{\circ}$ $\mathrm{tt}^{\circ} \Rightarrow^{\circ} f^{*}\left(\underline{x}^{\circ}, \mathrm{fst}_{f}(\underline{x})\right)={ }^{\circ}$ tt is true pointwise. The implication (2) $\left(f^{*}\left(\underline{x}^{\circ}, y^{\circ}\right)={ }^{\circ}\right.$ tt $\left.\Rightarrow^{\circ} \mathbf{s}_{f}(\underline{x})=\mathrm{tt}\right)(d)$ is true for all $d \in D$ covering $y$, hence for all $d \geq \xi(y)$, hence it is cofinally true. We deduce that $\mathbf{s}_{f}(\underline{x})$ is a faithful interpretation of the existential over $N^{*}:$ (3) $\mathbf{s}_{f}(\underline{x})={ }^{\circ}$ tt ${ }^{\circ}$ iff $f^{*}\left(\underline{x}^{\circ}, y^{\circ}\right)={ }^{\circ} \mathrm{tt}^{\circ}$ for some $y \in N^{*}$. By taking the contraposition of the implications (1) and (2) we obtain: (4) $\mathbf{s}_{f}(\underline{x})=^{\circ}$ $\mathrm{ff}^{\circ} \Rightarrow^{\circ} f^{*}\left(\underline{x}, \mathrm{fst}_{f}(\underline{x})\right)=^{\circ} \mathrm{ff}^{\circ}$ and $(5)\left(\mathrm{s}_{f}(\underline{x})={ }^{\circ} \mathrm{ff}^{\circ} \Rightarrow^{\circ} f^{*}(\underline{x}, y)={ }^{\circ} \mathrm{ff}\right)$. From (4), (5), if we replace $f$ by $\neg f$ we deduce: $(6) \mathbf{s}_{\neg f}(\underline{x})={ }^{\circ} \mathrm{ff}^{\circ}$ iff $\neg f^{*}\left(\underline{x}^{\circ}, y^{\circ}\right)={ }^{\circ} \mathrm{ff}^{\circ}$ for all $y \in N^{*}$. That is, all $f(\underline{x})$ is a faithful interpretation of the universal quantifier over $N^{*}:(7) \operatorname{all}_{f}(\underline{x})={ }^{\circ} \mathrm{tt}^{\circ}$ iff $f^{*}\left(\underline{x}^{\circ}, y^{\circ}\right)={ }^{\circ} t^{\circ}$ for all $y \in N^{*}$.

$\mathbf{s}_{f}$, fst $_{f}$ may be lifted to maps: $\mathbf{s}_{f}^{*}:\left(N^{*}\right)^{k} \rightarrow \mathcal{B}^{*}$, fst $_{f}^{*}:\left(N^{*}\right)^{k} \rightarrow \mathcal{B}^{*}$ having the same properties. Indeed, (1), (2), (4), (5) are true for all $\underline{x} \in\left(N^{*}\right)^{k}$ by Density, and, for all $\underline{x} \in\left(N^{*}\right)^{k},(3),(6),(7)$ follow from them. 
Lifting Recursive Maps to $N^{*}$. Maps on $N^{*}$ include a corresponding $f^{\circ}$ for each total recursive map $f$ over $N$. They are closed under composition. In order to prove they include all $\Delta_{2}^{0}$-maps, we only have to prove they are closed under minimalization. We first express what is minimalization in $N^{*}$. Fix any continuous property $f^{*}:\left(N^{*}\right)^{k}+1 \rightarrow \mathcal{B}^{*}$, any $\underline{x} \in\left(N^{*}\right)^{k}$. We say that $f^{*}$ is minimalizable on $\underline{x}$ iff $f^{*}(\underline{x}, y)$ is solvable in $N^{*}$ : for some $y \in N^{*}, f^{*}(\underline{x}, y)$ is true. We say that $f^{*}$ is minimalizable iff $f^{*}$ is minimalizable on all $\underline{x} \in\left(N^{*}\right)^{k}$. Let $g^{*}:\left(N^{*}\right)^{k} \rightarrow N^{*}$ be any continuous map. We say that $g^{*}$ is a minimalization of $f^{*}$ iff for all $\underline{x}, y \in$ $\left(N^{*}\right)^{k}+1, y=g^{*}(\underline{x})$ is the minimum solution to $f^{*}(\underline{x}, y)$. In other words, we ask the following statements to be true in $\mathcal{B}^{*}:\left(f^{*}\left(\underline{x}, g^{*}(\underline{x})\right)={ }^{\circ} \mathrm{tt}\right)$ and ${ }^{\circ}\left(y<g^{*}(\underline{x}) \Rightarrow^{\circ} f^{*}(\underline{x}, y)={ }^{\circ}\right.$ ff $)$. We say that maps over $N^{*}, D$ are closed under minimalization iff whenever a map $f^{*}$ is minimalizable, then there is some minimalization $g^{*}$ of $f^{*}$. It is immediate to show that minimalization, when it exists, it is unique up to extensional equality. We prove that, at least in the case $D=N$, maps over $N^{*}, D$ are closed under minimalization. Since all $N^{*, D}$ are recursively isomorphic, provided $D$ covers $N$, the thesis will follow for all D. We introduce a map $(\mu x . f)$ : $N^{k} \rightarrow N^{*}$, which is our candidate for $g$. We define, for all $\underline{x} \in\left(N^{*}\right)^{k}, d \in$ $N:(\mu x . f)(\underline{x})(d)=$ the minimum $x \leq d$ such that $f(\underline{x}, x)(\bar{d})=$ tt, if any, $d$ otherwise.

We will prove that if $f^{*}$ is minimalizable, then $(\mu x . f)^{*}$ is a minimalization of $f^{*}$. The first step is to check that $(\mu x . f)$ is, indeed, a map: $N^{k} \rightarrow N^{*}$. Before this, we show that, in the case $D=N$, we may weaken the requirement for being a stable map.

Lemma 15. (Cofinality and Well-Foundation). Choose $D=N$. Let $X$ be a recursive cofinal subset of $N$. Fix any sequence $L: D \rightarrow N$. Assume $>_{L}$ is well-founded when restricted to $X$. Then $L$ is stable.

Proof. We have to prove that all $d \in N$ are well-founded for $>_{L}$. Since $X$ is recursive cofinal, we may define a total recursive map $\phi: N \rightarrow N$ by: $\phi(x)=$ the first $y \in X$ such that $y \geq x$ in $N$. We will prove $d$ wellfounded for $>_{L}$ by principal induction over $\phi(d)$ in $\left(X,>_{L}\right)$, secondary induction over $\phi(d)-d$ (an integer $\geq 0$ by definition of $\phi$ ). Since wellfoundation is inductive, we have to check that all $e>_{L} d$ are well-founded w.r.t. $>_{L}$. We consider two cases, according if $e \leq \phi(d)$ or $e>\phi(d)$. Assume $e \leq \phi(d)$. Then $\phi(e)=\phi(d)$, but $(\phi(d)-d)>(\phi(e)-e) \geq 0$ because $\bar{e}>d$. Well-foundation of $e$ follows by secondary induction hypothesis. Assume $e>\phi(d)$. We will prove in this case that all $e^{\prime}>_{L} e$ are well-founded for $>_{L}$. Since well-foundation is inductive, it will follow $e$ well-founded. We have $\phi\left(e^{\prime}\right) \geq e^{\prime}>_{L} e>\phi(d)$, hence $\phi\left(e^{\prime}\right)>_{L} \phi(d)$ by the properties of $>_{L}$. By principal induction hypothesis, we conclude that all $e^{\prime}>_{L} e$ are well-founded for $>_{L}$, as wished.

We may now check that $(\mu x . f)$ is, indeed, a map: $N^{k} \rightarrow N^{*}$. We start with a general remark for all $y \in D^{*}$. 
Lemma 16. (Bar Lemma). Assume $P: D^{*} \rightarrow(D \rightarrow \mathcal{B})$ is defined by $P(y)(d)=(d \geq y(d))$, for all $y \in D^{*}, d \in D$. Then $P^{*}: D^{*} \rightarrow \mathcal{B}^{*}$, and $P^{*}(y)$ is true for all $y \in D^{*}$.

Proof. By Density, we only have to prove that $P^{*}\left(y^{\circ}\right) \in \mathcal{B}$, and that $P^{*}\left(y^{\circ}\right)$ is true for all $y \in D$. For all $d \in N, P^{*}\left(y^{\circ}\right)(d)$ unfolds to $(d \geq$ $\left.y^{\circ}(d)\right)$, that is, to $(d \geq y) . P^{*}\left(y^{\circ}\right)(d)$ may change value at most once, from $f f$ to $t \mathrm{t}$, hence it is stable. To prove that $P^{*}\left(y^{\circ}\right)$ is true, we just remark that it is stationary true (true for all $d \geq y$ ).

For instance, if $D=N$, then for all $y \in N^{*}=D^{*}$ we have $y(n) \leq n$ cofinally. Out of this remark we prove:

Lemma 17. (Convergence of $\mu x . f)$. Choose $D=N$. Let $f^{*}:\left(N^{*}\right)^{k}+$ $1 \rightarrow \mathcal{B}^{*}$ be any continuous property. If $f^{*}$ is minimalizable for all $\underline{x} \in N^{k}$, then $(\mu x . f): N^{k} \rightarrow N^{*}$

Proof. Abbreviate $g=(\mu x . f)$. Fix any $\underline{x} \in N^{k}$. By hypothesis, $f^{*}$ is minimalizable in $\underline{x} \in N^{k}$ : hence, for some $y \in N^{*}, f^{*}(\underline{x}, y)$ is true. Let $X=\left\{d \in D=N \mid f^{*}(\underline{x}, y)(d)=\operatorname{tt} \wedge y(d) \leq d\right\} . X$ is recursive, and cofinal in $D$, because both $f^{*}(\underline{x}, y)()=$. tt and $(y() \leq$.$) are stable booleans,$ and are true: the first one by assumption, the second one by 16 . By 15 , it is enough to prove that, for any $\underline{x} \in N^{k},>_{g(\underline{x})}$ is well-founded if restricted to $X$. As usual, we will in fact prove something more: for all $d, d_{1}, d_{2} \in X$, if $d_{1} \leq d_{2} \leq d$ and $y\left(d_{1}\right)=y\left(d_{2}\right)$, then $d$ is well-founded for $\left(X,>_{g(\underline{x})}\right)$. The thesis will follow by selecting $d=d_{1}=d_{2}$. We argue by: principal induction over $d_{1}$ and $>_{y}$, secondary induction over

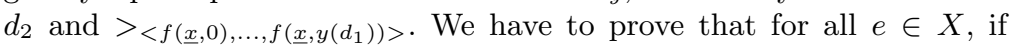
$e>_{g(\underline{x})} d$, then $e$ is well-founded in $X,>_{g(\underline{x})}$. Since $e>_{g(\underline{x})} d$, there are $d^{\prime}, e^{\prime}$ such that $d_{1}, d_{2} \leq d \leq d^{\prime} \leq e^{\prime} \leq e$ and $g(\underline{x})\left(d^{\prime}\right) \neq g(\underline{x})\left(e^{\prime}\right)$. In order to apply the inductive hypothesis, we have first to check: Claim. We cannot have: $f(\underline{x}, i)\left(d_{2}\right)=f(\underline{x}, i)\left(d^{\prime}\right)=f(\underline{x}, i)\left(e^{\prime}\right)$ for all $i \leq y\left(d_{2}\right)$. Proof of the Claim. We have $f\left(\underline{x}, y\left(d_{2}\right)\right)\left(d_{2}\right)=f^{*}(\underline{x}, y)\left(d_{2}\right)=\mathrm{tt}$, and $y\left(d_{2}\right) \leq d_{2} \leq d^{\prime}, e^{\prime}$, because $d_{2} \in X$. Hence for some $i_{0} \leq y\left(d_{2}\right) \leq d^{\prime}, e^{\prime}$ we have $f\left(\underline{x}, i_{0}\right)\left(d_{2}\right)=\mathrm{tt}$, while $f(\underline{x}, i)\left(d_{2}\right)=\mathrm{ff}$ for all $i<i_{0}$. Assume now $f(\underline{x}, i)\left(d_{2}\right)=f(\underline{x}, i)\left(d^{\prime}\right)=f(\underline{x}, i)\left(e^{\prime}\right)$ for all $i \leq i_{0} \leq y\left(d_{2}\right)$. Then $f\left(\underline{x}, i_{0}\right)\left(d^{\prime}\right)=f\left(\underline{x}, i_{0}\right)\left(e^{\prime}\right)=\mathrm{tt}$, while $f(\underline{x}, i)\left(d^{\prime}\right)=f(\underline{x}, i)\left(e^{\prime}\right)=\mathrm{ff}$ for all $i<i_{0} \leq d^{\prime}, e^{\prime}$. We conclude $g(\underline{x})\left(d^{\prime}\right)=g(\underline{x})\left(e^{\prime}\right)=i_{0}$, contradicting $g(\underline{x})\left(d^{\prime}\right) \neq g(\underline{x})\left(e^{\prime}\right)$. Proof of the Lemma, by cases over the Claim. We may now apply induction hypothesis to prove our thesis. Assume $y\left(d_{2}\right) \neq y\left(d^{\prime}\right)$, or $y\left(d^{\prime}\right) \neq y\left(e^{\prime}\right)$, or $y\left(e^{\prime}\right) \neq y(e)$. Since $d_{1} \leq d_{2} \leq d^{\prime} \leq e^{\prime} \leq e$, in all cases we deduce $e>y\left(d_{1}\right)$. Well-foundation of $e$ in $\left(X,>_{g(\underline{x})}\right)$ follows by principal induction hypothesis over the pair $e, e$. Assume $f(\underline{x}, i)\left(d_{2}\right) \neq f(\underline{x}, i)\left(d^{\prime}\right)$, or $f(\underline{x}, i)\left(d^{\prime}\right) \neq f(\underline{x}, i)\left(e^{\prime}\right)$ for some $i \leq y\left(d_{1}\right)=y\left(d_{2}\right)=y\left(d^{\prime}\right)=y\left(e^{\prime}\right)=y(e)$. Since $d_{2} \leq d^{\prime} \leq e^{\prime} \leq e$, in both cases we deduce $\left.e>_{\langle f}(\underline{x}, 0), \ldots, f\left(\underline{x}, y\left(d_{1}\right)\right)\right\rangle d_{2}$. Well-foundation of $e$ in $\left(X,>_{g(\underline{x})}\right)$ follows by secondary induction hypothesis over the pair $d_{1}, e$, and $y\left(d_{1}\right)=y(e)$. 
Lemma 18. (Minimalization Lemma). Choose $D=N$. Then maps over $N^{*, D}$ are closed under minimalization.

Proof. Let $f^{*}:\left(N^{*}\right)^{k+1} \rightarrow \mathcal{B}^{*}$ be any continuous property, and $g=$ $(\mu x . f)$. We have to show that, if $f^{*}$ is minimalizable for all $\underline{x} \in N^{k}$, then $g^{*}$ is a minimalization of $f^{*}$. We proved, in 3.4 , that $g^{*}$ is a continuous map. We have to prove that, for all $\underline{x}, y \in\left(N^{*}\right)^{k+1}$, the following statement is true in $\mathcal{B}^{*}:\left(f^{*}\left(\underline{x}, g^{*}(\underline{x})\right)={ }^{\circ}\right.$ tt $) \wedge^{\circ}\left(y<g^{*}(\underline{x}) \Rightarrow^{\circ} f^{*}(\underline{x}, y)=^{\circ}\right.$ $\mathrm{ff})$. By Density, it is enough to prove it for all $\underline{x}, y \in N^{k+1}$. We prove first the truth of the right-hand side, then the truth of the left-hand side. Right-hand side. It is true pointwise. Indeed, for all $\underline{x}, y \in N^{k+1}$ we have $(y<g(\underline{x})(d) \Rightarrow f(\underline{x}, y)(d)=\mathrm{ff})$, because $g(\underline{x})(d)$ is the smallest solution $z \leq n$ of $f(\underline{x}, z)(d)=\mathrm{tt}$, if any. Left-hand side. Since $f^{*}$ is minimalizable for all $\underline{x} \in N^{k}$, there is some $y \in N^{*}$ such that $\left(f^{*}(\underline{x}, y)=\mathrm{tt}\right)$. The thesis unfolds to: $(f(\underline{x}, g(\underline{x})(d))(d)=\mathrm{tt})$ holds cofinally on $d \in D$. For all $d \in D=N,(f(\underline{x}, g(\underline{x})(d))(d)=\mathrm{tt})$ is a consequence of $(n \geq y(d))$ and of $(f(\underline{x}, y(d))(d)=\mathrm{tt})$ : indeed, if there is some solution $z \leq n$ of $(f(\underline{x}, z)(d)=\mathrm{tt})$, then $(f(\underline{x}, g(\underline{x})(d))(d)=\mathrm{tt})$. Both $(n \geq \bar{y}()$. and $\left(f^{*}(\underline{x}, y)()=.\mathrm{tt}\right)$ are stable booleans and cofinally true, the first by 16 , the second by hypothesis. By the Filter Lemma 3, we conclude $\left(f^{*}\left(\underline{x}, g^{*}(\underline{x})\right)={ }^{\circ} \mathrm{tt}^{\circ}\right)$.

Out of the previous Lemmas, we deduce that continuous maps include $\Delta_{2}^{0}$-maps. Next step is to check that the reverse implication holds, and therefore continuous maps are exactly all maps recursive in the Halting problem (classically, all $\Delta_{2}^{0}$-maps). We also show that $\Delta_{2}^{0}$-maps may be obtained as the smallest class of maps definable using lifting of recursive maps, some suitable "some" maps, and minimalization.

Let $L \in N^{*}$. Then $\mathrm{L}$ is also a recursive map $L: D \rightarrow N$. Therefore it makes sense to consider its lifting $L^{\circ}: D^{*} \rightarrow N^{*}$. We say that $\delta \in D^{*}$ is a stationary point of $L^{\circ}$ on $D^{*}$ iff $\delta \leq^{\circ} \eta \Rightarrow^{\circ} L^{\circ}(\delta)={ }^{\circ} L^{\circ}(\eta)$ is true, for all $\eta \in D^{*}$. By Density, $\delta \in D^{*}$ is a stationary point of $L^{\circ}$ iff the implication above holds for $\eta=d^{\circ}$, and for all $d \in D$.

Lemma 19. (Stationarity Lemma). Let $L \in N^{*}$, and $L^{\circ}: D^{*} \rightarrow N^{*}$ be the lifting of $L$ (as a map: $D \rightarrow N$ ) to $D^{*}$. Assume $\delta \in D^{*}$ be a stationary point of $L^{\circ}$ on $D^{*}$. Then $L^{\circ}$ reaches its limit value in $\delta \in D^{*}: L={ }^{\circ} L^{\circ}(d)$ (equality between elements of $N^{*}$ ).

Proof. We have to prove that $\left.L(d)=L^{\circ}(\delta)(d)\right)$ holds cofinally on $d \in D$ : for every $d \in D$ there is some $e \geq d$ such that $\left.L(e)=L^{\circ}(\delta)(d)\right)=L(\delta(e))$. We argue by induction over $d$ and $>_{L}$. Define $\eta=j^{\circ}\left(d^{\circ}, \delta\right) \in D^{*}$. Then $\eta \geq^{\circ} \delta$ and $\eta \geq^{\circ} d^{\circ}$ are pointwise true. Since we assumed that $d$ is a stationary point, we deduce that $L^{\circ}(\delta)={ }^{\circ} L^{\circ}(\eta)$ is true. By definition of truth, $L(\delta(e))=L(\eta(e))$ for some $e \geq d$. By definition of $\eta$, we also have $\eta(e) \geq d$. We reason by cases. Assume both $L(\eta(e))=L(d)$ and $L(d)=L(e)$. We deduce $L(\delta(e))=L(\eta(e))=L(d)=L(e)$. We conclude there is some $e \geq d$ such that $L(e)=L(\delta(e))$. Assume instead either 
$L(\eta(e)) \neq L(d)$, or $L(d) \neq L(e)$. We deduce either $\eta(e)>_{L} d$, or $e>_{L} d$. If $\eta(e)>_{L} d$, we apply induction hypothesis over $\eta(e)$, if $e>_{L} d$, over $e$. We conclude there is some $e^{\prime} \in D$ such that $e^{\prime} \geq \eta(e) \geq d$, respectively, such that $e^{\prime} \geq e \geq d$, and such that, in both cases, $L\left(e^{\prime}\right)=L\left(\delta\left(e^{\prime}\right)\right)$.

Fix any recursive enumeration $\left\{d_{k}\right\}_{k} \in N$ of elements of $D$. Let $D(x)$ be the recursive predicate $(x \in D)$. Being a stationary point of $L$ is a property $P \in N \rightarrow \mathcal{B}^{*}$, defined by $\operatorname{stat}_{L}(x)=\operatorname{all}_{f}(x)$, with $f(x)(e)=$ $D(x) \wedge(D(y), x \leq y \Rightarrow L(x)=L(y))$, Then stat ${ }_{L}$ is a $\Delta_{2}^{0}$-map $: D \rightarrow$ $\mathcal{B}^{*}$, because it may be defined by composition of a negation, and of an existential over some decidable property. We already checked that universal quantifiers are faithfully interpreted in $N^{*}$. Thus, for all $x \in N^{*}$ $\operatorname{stat}_{L}(x)$ iff for all $y \in N^{*}, D^{\circ}(x) \wedge\left(D^{\circ}(y), x \leq{ }^{\circ} y \Rightarrow^{\circ} L^{\circ}(x)={ }^{\circ} L^{\circ}(y)\right)$. Using $D^{\circ}(z)$ iff $z={ }^{\circ} d$ for some $d \in D^{*}, D^{\circ}(y)$, we obtain: $\operatorname{stat}_{L}(x)$ iff $x={ }^{\circ} \delta$, for some $\delta \in D^{*}$, and for all $\eta \in D^{*},\left(\delta \leq{ }^{\circ} \eta \Rightarrow{ }^{\circ} L^{\circ}(\delta)={ }^{\circ} L^{\circ}(\eta)\right)$. That is: $\operatorname{stat}_{L}(x)$ iff for some $\delta \in D^{*}, x={ }^{\circ} \delta$ and $\delta$ is stationary for $L^{\circ}$ on $D^{*}$.

Theorem 20. (Characterization of continuous maps). Continuous maps are exactly $\Delta_{2}^{0}$-maps.

\section{Proof.}

$\Rightarrow$. By 18

$\Leftarrow$. Take any $f: N \rightarrow N^{*}$. Then $f(x)$ is a is total recursive map: $D \rightarrow N$, and $f(x)^{\circ}: D^{*} \rightarrow N^{*}$ is a $\Delta_{1}^{0}$-map. We will define a $\Delta_{2}^{0}$-map $s(x)=$ $\mu d$. stat $f(x)^{\circ}(d)$. By 18, $s(x)$ is defined provided $f(x)^{\circ}$ has some stationarity point. By 19, if $s(x)$ is always defined, then $f(x)={ }^{\circ} f(x)^{\circ}(s(x))$ for all $x$. The right-hand-side, being a composition of a $\Delta_{1}^{0}$-map with a $\Delta_{2}^{0}$-map, is a $\Delta_{2}^{0}$-map. All we have to prove is that there is some stationary point $d \in D^{*}$ for $f(x)^{\circ}$ on $D^{*}$. Some preliminaries. Fix any covering (Cov, $\xi)$, and any effective enumeration $\left\{d_{k}\right\}_{k}$ of elements of $D$. Let $x @ l$ denote the (integer code of) the concatenation of any integer $x$ with some list $l$ of integers, last $(l)$ denote the last element of a list $l$ (last $(l)=0$ if the list is empty)). Abbreviate $x \in \operatorname{Cov}(d)$ by $x \triangleleft d$. We will now define a map $g(x)(d)$, returning some finite succession of "possible positions" in which $\mathrm{f}(\mathrm{x})(\mathrm{d})$ might assume its limit value: $d_{k_{0}}, d_{k_{1}}, \ldots, d_{k_{n}}$. We ask that each next value in the list be a "better guess" for the point in which $\mathrm{f}$ assumes its limit value. That is, we want: $d_{k_{0}}<_{f(x)} d_{k_{1}}<_{f(x)} \ldots<_{f(x)} d_{k_{n}}$. We ask, besides, that $\left\{k_{1}, \ldots, k_{n}\right\} \subseteq \operatorname{Cov}(d)$, and that the last guess has no better guess indexed by some $i \triangleleft d$ (we ask that $d_{i}>_{f(x)} d_{k_{n}}$ for no $i \triangleleft d)$. We may recursively define some $g(x, k)(d)$ with the required property as follows: $g(x, k)(d)=d_{k}$ if there is no $h \triangleleft d$ such that $d_{h}>_{f(x)} d_{k}$, $g(x, k)(d)=d_{k} @ g(x, h)(d)$ for the first $h \triangleleft d$ such that $d_{h}>_{f(x)} d_{k}$, o.w. Then we set: $g(x)(d)=g(x, 0)(d)=$ some list $d_{k_{0}}, d_{k_{1}}, \ldots, d_{k_{n}}$ such that $\left\{k_{1}, \ldots, k_{n}\right\} \subseteq \operatorname{Cov}(d)$, and $d_{i}>_{f(x)} d_{k_{n}}$ for no $i \triangleleft d$.

We also set best $(x)(d)=\operatorname{last}(g(x)(d))=$ some $d_{k}$ such that $d_{i}>_{f(x)} d_{k}$ for no $i \triangleleft d$.

$g(x, k)(d)$ and best $(x)(d)$ are well-defined. We may prove that $g(x, k)(d)$ is well-defined for all $d \in D$ by induction w.r.t. the stable ordering $>_{f(x)}$. 
Indeed, we use the recursive call $g(x, k)(d)=g(x, h)(d)$ only when there is some $d_{h}>_{f(x)} d_{k}$, for some $h \triangleleft d$.

best $(x) \in N^{*}$. It is enough to prove that $g(x) \in N^{*}$, that is, that $>_{g(x)}$ is well-founded over $D$. Let us order the set of lists increasing under $<_{f(x)}$ lexicographically. Recall, we have $l>l^{\prime}$ lexicographically iff either $l$ is an extension of $l^{\prime}$, or for some list $l^{\prime \prime}$ and some integers $a<b$ (in the integer ordering) we have $l \geq l^{\prime \prime} a, l^{\prime} \geq l{ }^{\prime *} b$ (by prefix ordering). This set of list is well-founded because if $l$ is an extension of $l^{\prime}$ then last $(l)>_{f(x)} \operatorname{last}\left(l^{\prime}\right)$ because $l$ is increasing w.r.t. $>_{f(x)}$. Thus, last(.) is an increasing map from our set of lists and $D,>_{f(x)}$. We conclude that lexicographic ordering is well-founded.

Claim. The map $g(x)$ is weakly increasing w.r.t. the lexicographic ordering.

Proof of the Claim. Assume $e \geq d$ (in $D$ ), in order to prove $g(x)(e) \geq$ $g(x)(d)$ (lexicographically). Indeed, assume $e \geq d$. Let $r, s$ be the length of $g(x)(e), g(x)(d)$. Assume the two list are equal for all index $<r, s$. Then by induction on $s$, and the fact that $h \triangleleft d$ implies $h \triangleleft e$ for all $h \in N$, we may prove that the each element of $g(x)(d)$ is in $g(x)(e)$. Hence $g(x)(e)$ is an extension of $g(x)(d)$. Assume $g(x)(d), g(x)(e)$ are different in a first index $i<r, s$. Then the first $h \triangleleft e$ such that $d_{h}>_{f(x)} d_{i}$ is smaller than the first $h \triangleleft d$ such that $d_{h}>_{f(x)} d_{i}$, again because $h \triangleleft d$ implies $h \triangleleft e$.

Proof of $\operatorname{best}(x) \in N^{*}$. If $e>_{g(x)} d$, then for some $e \geq e^{\prime} \geq d^{\prime} \geq$ $d$ in $D$ we have $g(x)\left(e^{\prime}\right) \neq g(x)\left(d^{\prime}\right)$. Therefore $g(x)(e)>g(x)\left(e^{\prime}\right) \geq$ $g(x)\left(d^{\prime}\right)>g(x)(e)$ lexicographically, hence $g(x)(e)>g(x)(d)$ (again, lexicographically). Thus, $g(x)($.$) is an increasing map from >_{g}(x)$ and $D$ to some well-founded lexicographic ordering. We conclude that $>_{g}(x)$ is well-founded over $D$. Hence $g(x) \in N^{*}$.

best $(x)$ is a stationary point of $f(x)^{\circ}$. By definition, for all $i \triangleleft e$, if $d_{i} \geq$ best $(x)(e)$ in $D$, then $d_{i}>_{f(x)}$ best $(x)(e)$ is false, that is, $f(x)\left(d_{i}\right)=$ $f(x)$ (best $(x)(e)$ ). By unfolding definitions we obtain: (1) (best $(x) \leq{ }^{\circ}$ $\left.d_{i} \Rightarrow^{\circ} f(x)^{\circ}(\operatorname{best}(x))=f(x)^{\circ}\left(d_{i}\right)\right)(e)$ is true, for all $i \triangleleft e$. In particular, for all $d \in D,(1)$ is true for $e=j(d, x(i)) \geq d$, because $i \triangleleft x(i) \leq e$ and covering is monotone. Thus, (1) is cofinally true, hence true in $\mathcal{B}^{*}$, for all $i \in N$.

There are definition schemas for $\Delta_{2}^{0}$-maps, equivalent to minimalization, but easier to use on $N^{*}$ : for instance pointwise linear recursion. Fix any recursive well-founded relation $R$ on $N$. Assume $a: N^{*} \rightarrow \mathcal{B}^{*}, b(x)$ : $N^{*} \rightarrow N^{*}, c: N^{*} \rightarrow N^{*}$, and suppose that: $a(x) \Rightarrow^{\circ} R^{\circ}\left(c(x), x^{\circ}\right)$ is pointwise true for all $x \in N$. Then there is some (in fact, a unique) map $f$ solving: (LinRec) $f^{*}(x)=$ if $^{\circ}\left(a^{*}(x), b^{*}(x), f^{*}\left(c^{*}(x)\right)\right)$ for all $x \in N^{*}$. A continuous solution $f^{*}$ may obtained by solving the corresponding fixed point equation for recursive maps: $f(x)(d)=$ if $(a(x)(d), b(x)(d), f(c(x)(d))(d))$. We skip the proof of this result.

The last result of this section has an immediate proof, yet it is quite relevant. Let $\mathrm{g}$ be any recursive map: the theorem below says that if there is a limit solution $l \in N^{*} \operatorname{forg}^{\circ}(x)=0$, then $l$ may be effectively turned into a solution $x \in N$ of the same $g^{\circ}(x)=0$. From a logical viewpoint, this is a conservativity result: extending $N$ to $N^{*}$ adds no new solution to any equation. This justifies the interest of our model 
construction: we may use limit reasoning just to find integer solutions faster. From a topological viewpoint, the result is a density property (of $N$ in $N^{*}$ ): the set $O=\{x \mid g(x)=0\}$ is a basic open in effective discrete topology, and the theorem says that if $O$ intersects $N^{*}$, then $O$ intersects $N$.

Theorem 21. (Conservativity Theorem or Density Theorem). Let $g$ be any recursive map. If $g^{\circ}(l)=0$ for some $l \in N^{*}$, then $g(x)=0$ for some $x \in N$.

Proof. We have $g(l(d))=0$ cofinally in $D$, hence at least for some $d \in D$. Take $x=l(d) \in N$.

A last remark: $x$ cannot be, in general, the limit of $l$, because limits are not computable. The situation is similar when we get Real number by completion from Rational Numbers. Suppose we want to find square root of 2 with approximation $\epsilon=10^{-9}$, i.e., we want to find some $x \in Q$ such that $\left|x^{2}-2\right|<\epsilon$. This means that we have to find some inhabitant in the open set $O=\left\{x \mid x^{2} \in(2-\epsilon, 2+\epsilon)\right\}$. We know that $O$ is inhabited by $\sqrt{2}$ : since $Q$ is dense in $R$, this means that $O$ is inhabited by some $x \in Q$ (usually, we find some trunking $x$ of $\sqrt{2}$ ). The rational $x$ is always different from the irrational $\sqrt{2}$. The notation $\sqrt{2}$ is only used as a step in the computation whose output is $x$ : we are planning to use limit integers in the same way.

In the rest of the paper, we introduce a notion of realizer for equational statements in $N^{*}$. Let $\mathrm{f}$ be total recursive $: N \rightarrow N$. The constructive proof of $\forall l \in N^{*}: f^{\circ}(l)={ }^{\circ} 0$ is true implies $\exists x \in N$. $f(x)=0$ implicitly includes a (non-trivial) method to turn any proof of $f^{\circ}(l)={ }^{\circ} 0$, for some limit integer $l$, into the computation of an $x$ such that $f(x)=0$. In 5 , we explicitly describe such a method. The tool we use is the notion of realizer. A realizer represents, in form of an effective map, the construction implicit in an intuitionistic reasoning. In 6 , we will use realizers to constructivize one simple classical theorem requiring Excluded Middle on $\Delta_{2}^{0}$-formulas.

\section{A Realization interpretation for equations between limits}

In this section we introduce a notion of realizer for equations $t={ }^{\circ} u$, for $t, u \in N^{*}$. The we will show that classical equational reasoning about non-recursive maps on $N$ may be turned, first, into constructive reasoning about continuous maps on $N^{*}$, then, into operations building such realizers.

"Adherence maps" (or "realizers") of equations. Any equation $t={ }^{\circ} u$ is short for "the sequences $t, u \in N^{*}$ are topologically adherent", or $\forall d \in D . \exists e \geq \operatorname{d.t}(e)=u(e)$ From a constructive view point, this means that there is some effective way, given any $d \in D$, to find some $e \geq d$ such 
that $t(e)=u(e)$. We may represent such "effective way of computing $e$ out of $d$ " by some recursive map $\Phi: D \rightarrow D$ computing $e$. In this paper, we only use realizers for adherence statements w.r.t. Discrete Topology on $N$. For this reason, we will also call our realizers "adherence maps".

Definition 22. (Realization of equality). Let $\Phi: D \rightarrow D$ be any (total) recursive map. We call $\Phi$ an "adherence map" for $t={ }^{\circ} u$, and we write $\Phi: t={ }^{\circ} u$, if for all $d \in D, \Phi$ chooses some $\Phi(d) \geq d$ such that $t(\Phi(d))=u(\Phi(d))$.

$\Phi$ may be considered as the constructive content of topological adherence between limits: $\Phi$ is a "realizer" of $t={ }^{\circ} u$. If we do not have $\Phi$, in order to find some $e \in D$ such that $t(e)=u(e)$ we may just do blind search (we assumed $D$ effectively enumerated); if we do have $\Phi$, we have some more relevant information about computing $e \in D$.

We propose a realization semantic for closed equational reasoning over continuous functions $f^{*}, g^{*}$ on $N^{*}$ (we use no variables). For all $l \in N^{*}$, each logical rule $f_{1}^{*}(l)={ }^{\circ} g_{1}^{*}(l), f_{2}^{*}(l)={ }^{\circ} g_{2}^{*}(l), \ldots \vdash f^{*}(l)={ }^{\circ} g^{*}(l)$ will be interpreted an operation turning adherence maps $\Phi_{1}: f_{1}^{*}(l)={ }^{\circ} g_{1}^{*}(l)$, $\Phi_{2}: f_{2}^{*}(l)={ }^{\circ} g_{2}^{*}(l), \ldots$ for each hypothesis into an adherence map $\Phi$ : $f^{*}(l)={ }^{\circ} g^{*}(l)$ for the conclusion. Thus, in principle, adherence map would be automatically produced out of any classical reasoning on limits. They would be a kind of "microscope", singling out the construction hidden in the classical proof. Realization semantic may be used as follows. Let again $\mathrm{f}$ be total recursive $: N \rightarrow N$. Out of a classical proof of $f^{\circ}(l)={ }^{\circ} 0$, for some $l \in N^{*}$, we may automatically generate some $\Phi$ such that $\Phi: f(l)=0$. Then (by the Conservativity Theorem), we conclude $f(l(e))=0$ for all $d \in D$ and all $e=\Phi(d)$. Now $x=l(e)=l(\Phi(d))$, for any $d \in D$, is some integer solution for $f(x)=0$, computed using $f$, therefore using ideas from the original classical proof of $f(l)=0$.

The possibility of automatic generation, however, should not be taken too seriously. It may be really used only for short proof, in order to get an intuitive understanding of constructions hidden in classical reasonings. For long proofs, it takes less time to reformulate the goal constructively, in term of adherence map, and try prove it directly, rather than reusing the classical proof. Also in this case, however, the fact that some constructivization exists, both for the theorem and for all of its lemmas, it is of some help.

Definition 23. (Realization statements). Let $p: N^{k} \rightarrow N$ be any recursive map, $\Phi: D \rightarrow D$, and $\underline{t} \in\left(N^{*}\right)^{k}$. A realization statement is any statement of the form $\Phi: p^{\circ}(\underline{t})={ }^{\circ} t^{\circ}$, or just $\Phi: p^{*}(\underline{t})$ for short. We will sometimes drop the superscripts $(.)^{\circ},(.)^{*}$ for sake of readability.

Realization statements include previous realization statements $\Phi:\left(t={ }^{\circ}\right.$ $u)$, which may be expressed as $\left(t={ }^{\circ} u\right)={ }^{\circ}$ tt. We already remarked 
that statements of the form $p^{\circ}(\underline{t})$ are also closed under lifting to $\mathcal{B}^{*}$ of boolean operation (negation, conjunction, disjunction).

We introduce now semi-formal rules to derive statement of the shape $\Phi: p^{\circ}(\underline{t})$, then we will interpret them as operations on realizers $\mathrm{f}$.

Definition 24. (Equational Rules for $\Delta_{2}^{0}$-maps).

(1) (Atomic Rules). Let $p_{1}, \ldots, p_{k}, p$ be any total recursive properties of arity $h$. Then Atomic rules are all rules $p_{1}^{\circ}(\underline{l}), \ldots, p k^{\circ}(\underline{l}) \vdash p^{\circ}(\underline{l})$ (for $\left.\underline{l} \in\left(N^{*}\right)^{h}\right)$ such that $p_{1}(\underline{x}), \ldots, p k(\underline{x}) \vdash p(\underline{x})$ is true in $N$ for all $\underline{x} \in N^{h}$. (2) (Density of $N$ ). Let $q: N^{h+j} \rightarrow \mathcal{B}$. Given any $\underline{l} \in\left(N^{*}\right)^{h}$, and any recursive succession of proofs of $q^{\circ}\left(\underline{x}^{\circ}, \underline{m}\right)$, recursively indexed on $\underline{x} \in N^{h}$, we conclude $q^{\circ}(\underline{l}, \underline{m})$, for any $\underline{l} \in\left(N^{*}\right)^{h}$. This rules has infinitely many (recursively given) premises: $\ldots q^{\circ}\left(\underline{x}^{\circ}, \underline{m}\right) \ldots\left(\right.$ for all $\left.\underline{x} \in N^{k}\right) \vdash$ $q^{\circ}(\underline{l}, \underline{m})$

(3) (Some, First Axioms). Let $f$ be any total recursive property : $N^{h} \rightarrow$ $\mathcal{B}$, and $s(\underline{x})=\mathbf{s}_{f}(\underline{x}) \in\{\mathrm{tt}, \mathrm{ff}\}, c(\underline{x})=\mathrm{fst}_{f}(\underline{x}) \in N$. The "some" rule (an axiom) says that if $s(\underline{x})=\mathrm{ff}$, then $f(\underline{x}, y)=$ ff for all $y \in$ $N: \vdash\left(\neg^{\circ} s(\underline{x}) \Rightarrow^{\circ} \neg f(\underline{x}, y)^{\circ}\right)$ (all y $\left.\in N\right)$ while the "first" rule (another axiom) says that if $s(\underline{x})=\mathrm{tt}$, then $y=c(\underline{x})$ picks some solution of $f(\underline{x}, y)=$ tt $: \vdash\left(s(\underline{x}) \Rightarrow^{\circ} f^{\circ}\left(\underline{x}^{\circ}, c(\underline{x})\right)\right)$.

Atomic rules in point (1) includes, say $a \vee b, \neg a \vee b \vdash b$, that is, they include case reasoning and Cut rule. By combining (2) and (3), we may generalize (3) to any $\underline{l} \in\left(N^{*}\right)^{k}$ and $m \in N^{*}$ :

$\vdash \neg^{\circ} s^{*}(\underline{l}) \Rightarrow^{\circ} f^{\circ}(\underline{l}, m) \neq 0 \vdash s^{*}(\underline{l}) \Rightarrow^{\circ} f^{\circ}\left(\underline{l}, c^{*}(\underline{l})\right)=0$.

Out of (2) we deduce, instead, induction over $N^{*}$.

Lemma 25. (Induction Lemma). Let $\underline{m} \in\left(N^{*}\right)^{k}$, and $p$ be any total recursive property $: N^{k+1} \rightarrow \mathcal{B}$. Then $p^{*}$ satisfies the induction schema.

Proof. Assume $p^{\circ}\left(0^{\circ}, \underline{m}\right)$ and $p^{\circ}(l, \underline{m}) \Rightarrow^{\circ} p^{\circ}(l+1, \underline{m})$ for all $l \in N^{*}$. By induction over $N$ we deduce $p^{\circ}\left(x^{\circ}, \underline{m}\right)$ for all $x \in N$. By Density we conclude $p^{\circ}(l, \underline{m})$, for all $l \in N^{*}$.

Total recursive properties : $N \rightarrow \mathcal{B}^{*}$ also satisfy equivalent formulation of induction, like total induction. Merging of realization maps. Merging is an operation taking any sequence $\Phi_{1}: p_{1}(\underline{l}), \ldots, \Phi_{n}: p_{n}(\underline{l})$ of adherence maps, and returning an adherence map $\Phi: p_{1}(\underline{l}) \wedge \ldots \wedge p_{n}(\underline{l})$. Our Realization interpretation is parametric under such operation (which was already implicitly defined in the Filter Lemma 3 ). We include here a very particular case of merging, Left Merging, and we postpone the most general definition to the next paper. Merging is inspired by Coquand's game interpretation of Cut (see [2]). Left merging is functional, deterministic and sequential. General merging will be an imperative, non-deterministic protocol solving conflicts among concurrent processes.

Left Merging (two arguments). Assume $p_{1}, p_{2}: N^{k} \rightarrow \mathcal{B}$ be total recursive properties, $\underline{l} \in\left(N^{*}\right)^{k}$ be a vector of $k$ stable maps, and $\Phi_{1}: p_{1}(\underline{l}), \Phi_{2}$ : 
$p_{2}(\underline{l})$. That is: $\Phi_{1}(d) \geq d, \Phi_{2}(d) \geq d, p_{1}\left(\underline{l}\left(\Phi_{1}(d)\right)\right)=\mathrm{tt}, p_{2}\left(\underline{l}\left(\Phi_{2}(d)\right)\right)=\mathrm{tt}$ for all $d \in D$. We will define their left merging, which is some adherence map f s.t. $\Phi:\left(p_{1}(\underline{l}) \wedge p_{2}(\underline{l})\right)$, that is, $\Phi(d) \geq d, p_{1}(\underline{l}(f(d)))=\mathrm{tt}$, $p_{2}(\underline{l}(f(d)))=\mathrm{tt}$ for all $d \in D$. The left merging will be independent from $p_{1}, p_{2}$. The idea is to find some $\Phi(d) \geq d$ such that $l(\Phi(d))=\underline{l}\left(\Phi_{1}(d)\right)=$ $\underline{l}\left(\Phi_{2}(d)\right) . \Phi$ alternatively applies $\Phi_{1}, \Phi_{2}$ to $d_{0}=d: d_{0} \leq d_{1}=\Phi_{1}\left(d_{0}\right) \leq$ $d_{2}=\Phi_{2}\left(d_{1}\right) \leq d_{3}=\Phi_{1}\left(d_{2}\right) \leq d_{4}=\Phi_{2}\left(d_{3}\right) \leq \ldots$, until the value $\underline{l}(d)$ stops changing, that is, as long as we have: $l\left(d_{1}\right) \neq l\left(d_{2}\right) \neq \underline{l}\left(d_{3}\right) \neq$ $\underline{l}\left(d_{4}\right) \neq \ldots$ or, equivalently, as long as we have: $d_{1}<_{\underline{l}} d_{2}<_{\underline{l}} \underline{l} d_{3}<_{\underline{l}} d_{4}<_{\underline{l}}$ (1)

The result of $\Phi(d)$ is the first $d_{i}$, with $i \geq 2$, such that $\underline{l}\left(d_{i}\right)=\underline{l}\left(d_{i-1}\right) . f$ is well-defined because any recursive call is decreasing w.r.t. $>_{l}$. Besides, for some $d^{\prime}, d^{\prime \prime}$, we have $\underline{l}(f(d))=\underline{l}\left(d_{i}\right)=\underline{l}\left(d_{i-1}\right)=\underline{l}\left(\Phi_{1}\left(d^{\prime}\right)\right)=\underline{l}\left(\Phi_{2}\left(d^{\prime \prime}\right)\right)$ (because either $i$ is odd and $d_{i}=\Phi_{1}\left(d_{i-1}\right), d_{i-1}=\Phi_{2}\left(d_{i-2}\right)$, or $i$ is even and $\left.d_{i}=\Phi_{2}\left(d_{i-1}\right), d_{i-1}=\Phi_{1}\left(d_{i-2}\right)\right)$. We deduce: $p_{1}(\underline{l})(f(d))=$ $p_{1}\left(\underline{l}\left(\Phi_{1}\left(d^{\prime}\right)\right)\right)=0 \quad p_{2}(\underline{l})(f(d))=p_{2}\left(\underline{l}\left(\Phi_{2}\left(d^{\prime \prime}\right)\right)\right)=0$ We conclude that $\mathrm{f}$ is an adherence map.

Left Merging ( $n$ arguments). We repeatedly apply binary Left Merging, using left association of arguments (we merge the first two maps, the result with the third one and so forth). A more general choice for Merging will be considered in a forthcoming paper.

Definition 26. (Realization Semantic for $\Delta_{2}^{0}$-maps). For each logical rule, we will define now some adherence maps $\Phi: p(\underline{l})$ for conclusion, out of from adherence maps $\Phi_{1}: p_{1}(\underline{l}), \ldots, \Phi_{n}: p_{n}(\underline{l})$ for the hypothesis. Let $j(d, e) \geq d, e$ be any map picking some upper bound of $d, e$.

Case (1) (Atomic rules). We take $\Phi=$ left merging of $\Phi_{1}, \ldots, \Phi_{n}$. The map $\Phi$ realizes $p_{1}(\underline{l}) \wedge \ldots \wedge p_{n}(\underline{l})$, hence it realizes also $p(\underline{l})$, because, for all $d \in D, p_{1}(\underline{l}(d)) \wedge \ldots \wedge p n(\underline{l}(d))$ implies $p(\underline{l}(d))$.

Case (2) (Density of N). Assume $\Phi_{\underline{x}}: p^{\circ}(\underline{x}, \underline{m})$, recursively on $\underline{x}$. This means $p^{\circ}(\underline{x}, \underline{m})(f \underline{x}(d))=0$ for all $d$. We define a map $\Phi$ such that $p^{\circ}(\underline{l}, \underline{m})(\Phi(d))=0$ for all $\underline{l} \in N^{k}$. Such a $\Phi$ repeatedly applies $d \mapsto$ $\Phi_{l(d)}(d)$, starting from $d_{0}=d: d_{0} \leq d_{1}=\Phi_{l(d)}\left(d_{0}\right) \leq d_{2}=\Phi_{l\left(d_{1}\right)}\left(d_{1}\right) \leq$ $\left.d_{3}=\Phi_{l\left(d_{2}\right)}\left(d_{2}\right) \leq d_{4}=\Phi_{l\left(d_{3}\right)}\left(d_{3}\right)\right) \leq \ldots$, until the value $\underline{l}(d)$ stops changing, that is, as long as we have: $\underline{l}\left(d_{0}\right) \neq \underline{l}\left(d_{1}\right) \neq \underline{l}\left(d_{2}\right) \neq \underline{l}\left(d_{3}\right) \neq \underline{l}\left(d_{4}\right) \neq \ldots$ or, equivalently, as long as we have:

$d_{1}<_{\underline{l}} d_{2}<_{\underline{l}} d_{3}<_{\underline{l}} d_{4}<_{\underline{l}} \ldots$..

Formally, $\Phi$ is recursively defined by: $\Phi(d)=\Phi_{\underline{l}(d)}(d)$ if $\underline{l}\left(\Phi_{\underline{l}(d)}(d)\right)=$ $\underline{l}(d)$, and $\Phi(d)=f\left(f_{l(d)}(d)\right)$ if $\underline{l}\left(\Phi_{l(d)}(d)\right) \neq \underline{l}(d)$. The result of $\Phi(d)$ is the first $d_{i}$ (with $i \geq 1$ ) such that $\underline{l}\left(d_{i}\right)=\underline{l}\left(d_{i-1}\right) . f(d)$ is well-defined by induction over $>_{\underline{l}}$. For all $i \in N$, just by definition unfolding we get $p\left(\underline{l}\left(d_{i-1}\right), \underline{m}\left(d_{i}\right)\right)=$ tt. Indeed, if we set $\underline{x}=\underline{l}\left(d_{i-1}\right)$, then from definition of $d_{i}$ and $\Phi_{\underline{x}}(d)$ we deduce: $p\left(\underline{l}\left(d_{i-1}\right), \underline{m}\left(d_{i}\right)\right)=p(\underline{x}, \underline{m})\left(d_{i}\right)=$ $p(\underline{x}, \underline{m})\left(\Phi_{\underline{l}}(d(i-1))\left(d_{i-1}\right)\right)=p(\underline{x}, \underline{m})\left(\Phi_{\underline{x}}\left(d_{i-1}\right)\right)=\mathrm{tt}$. We may now check that $p^{\circ}(\underline{l}, \underline{m})(\Phi(d))=0$, for all $d \in D: p^{\circ}(\underline{l}, \underline{m})(\Phi(d))=$ (by definition of $f) p^{\circ}(\underline{l}, \underline{m})\left(d_{i}\right)=$ (by definition of lifting) $p\left(\underline{l}\left(d_{i}\right), \underline{m}\left(d_{i}\right)\right)=\left(\right.$ by $\underline{l}\left(d_{i}\right)=$ $\left.\underline{l}\left(d_{i-1}\right)\right) p\left(\underline{l}\left(d_{i-1}\right), \underline{m}\left(d_{i}\right)\right)=$ (by the remark above)tt.

Case (3) (Some, First). Let $m=<f, \underline{x}>\in N$ be the name of to the map $f(\underline{x},$.$) . Then \mathbf{s}_{f}(\underline{x})$ has been defined in term of the covering $\triangleleft_{m}, \xi_{m}$, the covering number $m$ in our list. 
(Some). Fix any "some" axiom $\neg \mathbf{s}_{f}(\underline{x}) \Rightarrow f(\underline{x}, y) \neq 0\left(\underline{x} \in N^{k}, y \in N\right)$. Define $f(d)=j(d, e) \in D$, with $e=\xi_{m}(y)$. Then $y \triangleleft_{m} \Phi(d)(\Phi(d)$ covers $y)$. By definition of $\mathbf{s}_{f}(\underline{x})$, if $\neg s_{f}(d)(\underline{x})$ then no point $z$ covered by $\Phi(d)$ solves $f(\underline{x}, z)=0$. We conclude: $\neg \mathbf{s}_{f}(d)(\underline{x}) \Rightarrow^{\circ} f(\underline{x}, y)=\mathrm{ff}$.

(First). We just take $\Phi=$ id. We have to prove that id is an adherence map for any "first" axiom, i.e., that the statement $\left(s_{f}(\underline{x}) \Rightarrow^{\circ}\right.$ $f\left(\underline{x}\right.$, fst $\left.\left._{f}(\underline{x})\right)=\mathrm{tt}\right)(d)$ is true for all $d \in D$. But we already checked it is pointwise true.

In this section we defined a translation from classical proofs to programs. In the next section, inspired by this translation, we will constructivize, for some classical reasoning requiring Excluded Middle on $\Delta_{2}^{0}$-formulas (formulas without nested quantifiers).

\section{One example of constructivization of $\Delta_{2}^{0}$-classical reasoning}

In this section we will consider a simple combinatorial result over $\mathrm{N}$, Minimum Principle, requiring Excluded Middle over $\Delta_{2}^{0}$-formulas (onequantifier formulas). Our goal is to prove them constructively over $N^{*}$. The constructive proof in this section was originally obtained mechanically, by applying our Realizability Interpretation on classical reasoning. For sake of simplicity, however, we will include here no details, only a few hints, of how the constructive proof was obtained.

In fact, in further examples (not included here) Realization Semantic will only be used to ensure that some constructive version of a classical result proved by $\Delta_{2}^{0}$-Excluded Middle exists (provided we shift from $N$ to $N^{*}$ ). It usually takes too long to use Realization as an effective method to automatically produce a constructive proof out of some classical proof. Fix any $D$ equipped with some covering $(\triangleleft, \xi)$ of $N$. Let $\operatorname{Cov}(d)=$ the finite set of points covered by $d=\{y \in N \mid y \triangleleft d\}$. For sake of simplicity, in this section we assume that there is some $d \in D$ covering no points, i.e. with $\operatorname{Cov}(d)=\emptyset$; that the set covered by $j(d, e) \in D$ is the union of the sets covered by $d, e \in D$, i.e. $\operatorname{Cov}(j(d, e))=\operatorname{Cov}(d) \cup \operatorname{Cov}(e)$; and that $\xi(x) \in D$ covers exactly $\{x\}: \operatorname{Cov}(\xi(x))=\{x\}$. The reader might think of $D=\mathcal{P}_{\text {fin }}(N)$ as example.

Lemma 27. (Minimum Principle for integer functions). Classically, for each (total recursive) $f: N \rightarrow N$ there is some minimum point $m \in N$. $m$ is a minimum point for $f$ iff $f(m) \leq f(y)$ for all $y \in N$.

Proof (Classical). Start with $x_{0}=0 \in N$ (some arbitrary choice). If for all $y \in N$ we have $f\left(x_{0}\right) \leq f(y)$, we may choose $m=x_{0}$. Suppose instead $f\left(x_{0}\right)>f(y)$ for some $y$. Let $x_{1}=$ the first such $y$. If for all $y \in N$ we have $f\left(x_{1}\right) \leq f(y)$, we may choose $m=x_{1}$. Suppose instead $f\left(x_{1}\right)>f(y)$ for some $y$. Let $x_{2}=$ the first such $y$. If we continue in this way, we produce 
a sequence: $f\left(x_{0}\right)>f\left(x_{1}\right)>f\left(x_{2}\right)>f\left(x_{3}\right)>f\left(x_{4}\right)>\ldots$ of length at most $f\left(x_{0}\right)$. After at most $f\left(x_{0}\right)$ steps, we find some $m=x_{i}$ such that for all $y \in N$ we have $f\left(x_{i}\right) \leq f(y)$.

The minimum $m$ as a stable map $m \in N^{*}$. The classical proof of Minimum Principle defines $m$ using a suitable "some" map $s(x)=\exists y \cdot(f(x)>$ $f(y)$ ). Indeed, we may formally define $m=\operatorname{out}(0)$, with out $(x)=x$ if $f(x) \leq f(y)$ for all $y \in N$, o.w. out $(x)=\operatorname{out}(y)$ for the first $y \in N$ such that $f(y)<f(x)$ (The choice of the first point is just some arbitrary choice). Thus, $\mathrm{m}$ is a $\Delta_{2}^{0}$-map on the recursive code of $f$. Using our model of $\Delta_{2}^{0}$-maps, we may turn the classical definition of $m \in N$ into the definition of a stable map $m \in N^{*}$. We only have to relativize the "out" map to the points covered by some $d \in D$. We set $m(d)=\operatorname{out}(0)(d)$, with out $(x)(d)=x$ if $f(x) \leq f(y)$ for all $y \triangleleft d$, o.w. out $(x)(d)=\operatorname{out}(y)(d)$ for the first point $y \triangleleft d$ such that $f(y)<f(x)$. $m$ is defined using pointwise linear recursion over the value of $f(x)$, because in the case out $(x)(d)=\operatorname{out}(y)(d)$ we must have $f(y)<f(x)$. A simple characterization of $m \in N^{*}$ : out $(0)(d)$ produces a sequence: $f(0)=$ $f\left(x_{0}\right)>f\left(x_{1}\right)>f\left(x_{2}\right)>f\left(x_{3}\right)>f\left(x_{4}\right)>\ldots$ with $x_{1}, x_{2}, \ldots, x_{n} \triangleleft d$. Then $m(d)$ in fact produces the (first) minimum point of $f$ on $\operatorname{Cov}(d) \cup 0$. $m(d)=$ the first minimum point of $f$ on $\operatorname{Cov}(d) \cup\{0\}$.

A constructive Realization of the Minimum Principle. The constructive interpretation of " $f(m) \leq f(y)$ for all $y \in N$ ", in our Realization Semantic, is: $\Phi:(f(m) \leq f(y))$. A realizer $\Phi$ of the statement $(f(m) \leq f(y))$ can be defined by $f(d)=j(d, \xi(y))=$ some $e \geq d$ covering $y$. In order to check $\phi: f(m) \leq f(y)$ we have to prove $f(m(e)) \leq f(y)$ for $e=\phi(d)$. This follows by the fact $m(e)=\operatorname{minimum}$ of $f$ on $\operatorname{Cov}(e)$, and $y \in \operatorname{Cov}(e)$. Discussing the constructive Minimum Point Principle. We briefly compare the classical minimum principle with its constructive version. Instead of finding a minimum point $m \in N$ for $f$ on $N$, we find a minimum $m(d) \in N$ of $f$ on $\operatorname{Cov}(d) \cup\{0\}$, some finite set. All we do know is $f(m(d)) \leq f(y)$, for $y \in \operatorname{Cov}(d)$ or $y=0$. For a generic $y \in N$, we cannot guarantee $f(m(d)) \leq f(y)$. Yet, we may force $f(m(d)) \leq f(y)$ to be true by enlarging d to some $e=\phi(d)=j(d, \xi(y))$, covering also $y$. By the assumptions of this section, $\operatorname{Cov}(e)=\operatorname{Cov}(d) \cup\{y\}$. Adding the point y may change the value of $m(d)$. Indeed, if $f(m(d))<_{f}(y)$, or $f(m(d))=f(y)$ but $m(d) \leq y$ thenm $(d)$ is the first minimum of $f$ also in $\operatorname{Cov}(e) \cup\{y\}$ and $m(e)=m(d)$; if $f(m(d))>_{f}(y)$, or $f(m(d))=f(y)$ and $m(d)>y$, the first minimum is $y$, and $m(e)=y$. Thus, the "minimum" we get is a flow $\underline{m}(d)$ of values indexed over $d \in D$, rather than a single value. The key point to remark is that $m(d)$ is stable on $d$ : the successions of all possible changes of $m(d)$ form a well-founded tree. Indeed, whenever $m(d)$ changes then either $f(m(d)) \leq f(0)$ decreases, and it may decrease at most $f(0)$ times, or $f(m(d))$ stays the same, but $m(d)$ decreases: thus, the tree of all possible changes is well-founded.

By the Conservativity Theorem, whenever we use the classical Minimum Principle in order to prove the existence of some integer with some decidable property, we may use the constructive version of it in order to define some effective way to get such an integer. The following example is due to T. Coquand: there is some $x$ such that $f(x) \leq f(g(x))$. 
The classical proof is immediate: take $x=$ the minimum point $m$ of $f$. Since $f(m) \leq f(y)$ for all $y \in N$, in particular we have $f(m) \leq f(g(m))$. We may define an effective way of finding such an $m$ in our Realization Semantic.

Let $l=g(m)$. Then we have to define a realizer $\Phi:(f(m) \leq f(l))$ from a family of realizers $\Phi_{y}:(f(m) \leq f(y))$ In the previous paragraph we already defined $\Phi_{y}(d)=j(d, \xi(y)): \Phi_{y}$ adds the point y to the points covered by $d$. We use the method outlined in the proof of the Density rule. We start from any $d$, and we keep on applying $d \mapsto f_{l(d)}(d)$, starting from $d_{0}=d: d_{0} \leq d_{1}=f_{l}\left(d_{0}\right)\left(d_{0}\right) \leq d_{2}=\Phi_{l}\left(d_{1}\right)\left(d_{1}\right) \leq d_{3}=\Phi_{l}\left(d_{2}\right)\left(d_{2}\right) \leq \ldots$ until the value $l(d)=g(m(d))$ stops changing, that is, as long as we have: $g\left(m\left(d_{0}\right)\right) \neq g\left(m\left(d_{1}\right)\right) \neq g\left(m\left(d_{2}\right)\right) \neq g\left(m\left(d_{3}\right)\right) \neq g\left(m\left(d_{4}\right)\right) \neq \ldots$

A solution to $f(x) \leq f(g(x))$. No matter what $d_{i}$ is, when the succession $d_{0}, d_{1}, d_{2}, \ldots$ stops in $d_{i}$, we find a solution to $f(x) \leq f(g(x))$. Let us analyze which solution we get, assuming we start from some $d_{0}$ covering no points: $\operatorname{Cov}\left(d_{0}\right)=\emptyset$. Then $m\left(d_{0}\right)=$ the minimum of $f$ on $\{0\}=0$. By definition of $d_{i}=f_{l(d(i-1))}\left(d_{i}\right), d_{i}$ covers the points covered by $d_{i-1}$, plus $g\left(m\left(d_{i-1}\right)\right)$. By induction on $i$, we may prove: $\operatorname{Cov}\left(d_{i}\right)=\left\{g\left(m\left(d_{0}\right)\right), \ldots, g\left(m\left(d_{i-1}\right)\right)\right\}$.

Besides, if $g\left(m\left(d_{i}\right)\right)$ changes, then $m\left(d_{i}\right)$ changes, and by definition of $m, m\left(d_{i}\right)$ becomes $g\left(m\left(d_{i}-1\right)\right)$, the only new point covered by $d_{i}$. Thus, $m\left(d_{i}\right)=g\left(m\left(d_{i-1}\right)\right)=g\left(g\left(m\left(d_{i-2}\right)\right)\right)=g^{i}\left(m\left(d_{0}\right)\right)=g^{i}(0)$ and

$\operatorname{Cov}\left(d_{i}\right)=\left\{g^{0}(0), \ldots, g^{i-1}(0)\right\}$

The succession $g^{0}(0), g^{1}(0), g^{2}(0), \ldots$ continues as long as we have: $f\left(g^{i}(0)\right)>$ $f\left(g^{i+1}(0)\right)$ or $f\left(g^{i}(0)\right)=f\left(g^{i+1}(0)\right)$ but $g^{i}(0)>g^{i+1}(0)$ (This last clause is rather arbitrary, it is just there because we want $m(d)$ to be the first minimum of $f$ on $\left.\left\{g^{0}(0), g^{1}(0), g^{2}(0), ..\right\}\right)$. When we stop, we have $f\left(g^{i}(0)\right) \leq f\left(g^{i+1}(0)\right)$, and we found some $x=g^{i}(0)$ such that $f(x) \leq f(g(x))$.

Conclusion (of the section). Compare this method of find some $\mathrm{x}$ such that $f(x) \leq f(g(x))$ with the only method we usually associate to a classical proof of $\exists x . f(x) \leq f(g(x))$ : blind search through $x=0,1,2, \ldots$. In the first case, we only look through the values $x=g^{0}(0), g^{1}(0), g^{2}(0), \ldots$. In the second one, we look through all possible values of $x$. It is curious to remark, however, that the search through $x=g^{0}(0), g^{1}(0), g^{2}(0), \ldots$, was in fact implicit in the classical proof, yet it was invisible until we used Realization Semantic to make it explicit. Realization Semantic is a kind of microscope, singling out constructive features deeply hidden in classical reasoning.

We end this section outlining a canonical choice for the direct set D. We propose the choice $D=\mathcal{P}_{\text {fin }}(N)<\omega$ (finite sequences over $\mathcal{P}_{\text {fin }}(N)$ ), for efficiency reasons we will explain.

The choice of the direct set D. We said D is intended to represent the computation state. More specifically, we want $d \in D$ to be a data structure, storing all numerical instances $y \in N$ of the "some" axiom: $\neg s(\underline{x}) \Rightarrow^{\circ} \neg f(\underline{x}, y)^{\circ}\left(\underline{x} \in N^{k}, y \in N\right)$ which are really used by a classical proof of some $p^{\circ}(\underline{l})$. Let $m=<_{f}, \underline{x}>$ be the name of this particular instance of the "some" axiom. We interpret $y \triangleleft_{m} d$ by:

"at computation stage $d$, we used the instance $y \in N$ of the "some" axiom named $m$ " 
For this reason, in the definition both of $s_{d}(\underline{x}), c_{d}(\underline{x})$, and associated realizers we considered only the set of integers covered by the $m$-th component of $d$. Having all coverings $\left(\triangleleft_{n}, \xi_{n}\right)$ for $n \in N$ equal to the same is conceptually correct, but quite rough: in this way, we pile together integers used to instantiate different "some" axioms. If all covering are the same, whenever we compute $s_{d}(\underline{x})$, that is, we decide if $f(\underline{x}, y)=\mathrm{tt}$ for some $y \triangleleft_{m} d$, we are in fact searching through all $y \triangleleft_{n} d$ for some $n \in N$. Computation of $s_{d}(\underline{x})$ slows down considerably. For this reason, we choose $D=\mathcal{P}_{\text {fin }}(N)<\omega$, the set of all sequences $J=\left\{J_{n}\right\}_{n \in \omega}$ of finite subsets of $N$, such that $J_{n}=\emptyset$ from some $n$ on. We order $D$ by pointwise inclusion: $J \subseteq H$ iff $J_{n} \subseteq H_{n}$ for all $n$. We will denote by $\emptyset$ the bottom of $D$ (the sequence always empty). We define $j=$ l.u.b., that is, we set $j(J, H)_{n}=J_{n} \cup H_{n}$ (for all $\left.n \in N\right)$. We define $y \triangleleft_{m} J$ ( $y$ is covered by the $m$-th component of $J$ ) by $\left(y \in J_{m}\right)$, and $\xi_{m}(x)$ (the canonical $J$ whose $m$-th component covers $x$ ) by the smallest $J$ which $m$-covers $\mathrm{x}$. Such a $J$ is defined by: $J_{n}=\{x\}$ if $n=m, J_{n}=\emptyset$ if $n \neq m$

\section{References}

[0] C. J. Ash and J. F. knight, "Computable Structures and the Hyperarithmetical Hierarchy", Elsevier, 20

[1] S. Berardi. "A Model for $\Delta_{2}^{0}$-maps within Intuitionistic Arithmetic". Technical Report, Torino University, 2004.

[2] T. Coquand. "A Semantic of Evidence for Classical Arithmetic", Journal of Symbolic Logic, 60-1 (1995), pp. 325-337.

[3] S. Hayashi, Masahiro Nakata. "Limiting First Order Realizability Interpretation", Submitted to: Sci. Math. Japonicae. Vol 53, No. 1 (2000), 101-103. 